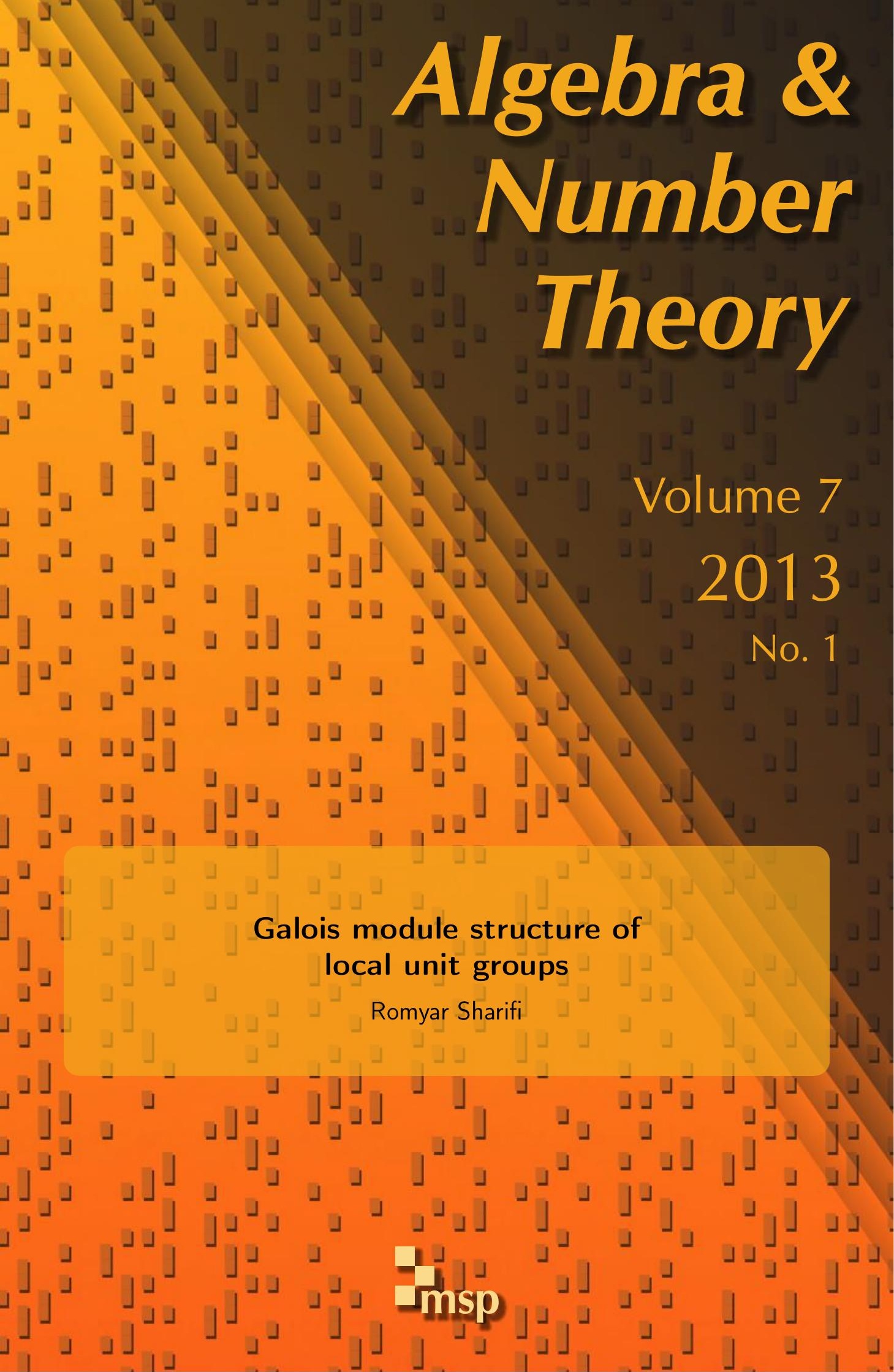




\title{
Galois module structure of local unit groups
}

\author{
Romyar Sharifi
}

\begin{abstract}
We study the groups $U_{i}$ in the unit filtration of a finite abelian extension $K$ of $\mathbb{Q}_{p}$ for an odd prime $p$. We determine explicit generators of the $U_{i}$ as modules over the $\mathbb{Z}_{p}$-group ring of $\operatorname{Gal}\left(K / \mathbb{Q}_{p}\right)$. We work in eigenspaces for powers of the Teichmüller character, first at the level of the field of norms for the extension of $K$ by $p$-power roots of unity and then at the level of $K$.
\end{abstract}

\section{Introduction}

Fix an odd prime $p$ and a finite unramified extension $E$ of $\mathbb{Q}_{p}$. We use $F_{n}$ to denote the field obtained from $E$ by adjoining to $E$ the $p^{n}$ th roots of unity in an algebraic closure of $\mathbb{Q}_{p}$. The $i$ th unit group in the unit filtration of $F_{n}$ will be denoted by $U_{n, i}$. The object of this paper is to describe generators of the groups $U_{n, i}$ as modules over the $\mathbb{Z}_{p}$-group ring of $G_{n}=\operatorname{Gal}\left(F_{n} / \mathbb{Q}_{p}\right)$. We express these generators in terms of generators of the pro- $p$ completion $D_{n}$ of $F_{n}^{\times}$as a Galois module. In fact, one consequence of our work is a rather elementary proof of an explicit presentation of $D_{n}$ as such a module, as was proven by Greither [1996] using Coleman theory.

Instead of working with all of $D_{n}$ at once, we find it easier to work with certain eigenspaces of it. For this and several other purposes, it will be useful to think of the Galois group $G_{n}$ as a direct product of cyclic subgroups

$$
G_{n}=\Delta \times \Gamma_{n} \times \Phi,
$$

where $\Delta \times \Gamma_{n}=\operatorname{Gal}\left(F_{n} / E\right)$ with $|\Delta|=p-1$ and $\left|\Gamma_{n}\right|=p^{n-1}$, and $\Phi$ is isomorphic to $\operatorname{Gal}\left(E / \mathbb{Q}_{p}\right)$. We then decompose $D_{n}$ into a direct sum of $p-1$ eigenspaces for powers of the Teichmüller character $\omega: \Delta \rightarrow \mathbb{Z}_{p}^{\times}$. For any integer $r$, the $\omega^{r}$-eigenspace $D_{n}^{(r)}$ of $D_{n}$ is the subgroup of elements upon which $\sigma \in \Delta$ acts by left multiplication by $\omega(\sigma)^{r}$. This definition depends only on $r$ modulo $p-1$, so we fix $r$ with $2 \leq r \leq p$. Note that $D_{n}^{(r)}$ is a module over the group ring $A_{n}=\mathbb{Z}_{p}\left[\Gamma_{n} \times \Phi\right]$.

Supported in part by an NSF Postdoctoral Research Fellowship, an NSERC Discovery Grant, the Canada Research Chairs program and NSF award DMS-0901526.

MSC2010: 11SXX.

Keywords: Galois module structure, unit filtration, local field. 
In fact, as we shall see in Section 3.1, the $A_{n}$-module $D_{n}^{(r)}$ has a generating set with just one element if $r \leq p-2$, three elements if $r=p-1$, and two elements if $r=p$.

We will be interested in the $A_{n}$-module structure of the groups $V_{n, i}^{(r)}=D_{n}^{(r)} \cap U_{n, i}$. It turns out that

$$
V_{n, i}^{(r)} \supsetneq V_{n, i+1}^{(r)}=V_{n, i+2}^{(r)}=\cdots=V_{n, i+p-1}^{(r)}
$$

for all $i \equiv r \bmod p-1$ (see Lemma 2.1), so we will consider only such $i$ and set $V_{n, i}=V_{n, i}^{(r)}$.

Our main results, Theorems 4.3.1 and 4.3.3, provide a small set of at most $n+1$ generators of $V_{n, i}$ as an $A_{n}$-module and state that any proper generating subset of it has cocardinality 1 . The elements of this set are written down explicitly as $A_{n}$-linear combinations of elements of the generators of $D_{n}^{(r)}$. In Section 4.2, elements of a special form are constructed so as to lie as deep in the unit filtration as possible. In Section 4.3, these are refined to elements of the same form that instead lie just deep enough to be in $V_{n, i}$, which are in turn the generators that we use.

It is convenient to work first in the field of norms $F$ of Fontaine-Wintenberger for the tower of extensions $F_{n}$ of $E$. This is a field of characteristic $p$, the multiplicative group of which is the inverse limit of the $F_{n}^{\times}$. We prove analogues of all of the abovementioned results first at this infinite level, prior to applying them in descending to the level of $F_{n}$. The fact that the $p$ th power map is an automorphism of $F^{\times}$ simplifies some of the computations. Moreover, the structure of the eigenspaces of the pro- $p$ completion of $F^{\times}$, which we study in Section 3.1, is somewhat simpler than that of the $D_{n}^{(r)}$. We construct special elements in the eigenspaces of the groups in the unit filtration in Section 3.2, refine them in Section 3.3, and prove generation and a minimality result in Section 3.4.

We see a number of interesting potential applications for the results of this paper. To mention just one, it appears to make possible the computation of the conductors of all degree $p^{n}$ Kummer extensions of $F_{n}$ in terms of the Kummer generator of the extension. The problem of making this computation, which was approached by the author in three much earlier papers, has until now seemed beyond close reach in this sort of generality.

\section{Preliminaries}

We maintain the notation of the introduction and introduce some more. Recall from [Wintenberger 1983] that the field of norms $F$ for the extension $F_{\infty}=\bigcup_{n} F_{n}$ of $E$ is a local field of characteristic $p$ with multiplicative group

$$
F^{\times}=\lim _{\longleftarrow} F_{n}^{\times},
$$

the inverse limit being taken with respect to norm maps. 
Let $\zeta=\left(\zeta_{p^{n}}\right)_{n}$ be a norm compatible sequence of $p$-power roots of unity, with $\zeta_{p^{n}}$ a primitive $p^{n}$ th root of unity in $F_{n}$. Then $\lambda=1-\zeta=\left(1-\zeta_{p^{n}}\right)_{n}$ is a prime element of $F$.

For $m \geq n$, let $N_{m, n}: F_{m} \rightarrow F_{n}$ be the norm map. Recall that the addition on $F$ is given by

$$
(\alpha+\beta)_{n}=\lim _{m \rightarrow \infty} N_{m, n}\left(\alpha_{m}+\beta_{m}\right)
$$

for $\alpha=\left(\alpha_{n}\right)_{n}$ and $\beta=\left(\beta_{n}\right)_{n}$ in $F$. We fix an isomorphism of the residue field of $E$ (and thereby each $F_{n}$ ) with $\mathbb{F}_{q}$, with $q$ the order of the residue field. Using this, the field $\mathbb{F}_{q}$ is identified with a subfield of $F$ via the map that takes $\xi \in \mathbb{F}_{q}^{\times}$to $\left(\tilde{\xi}^{p^{-n}}\right)_{n} \in F^{\times}$, where $\tilde{\xi}$ is the $(q-1)$ st root of unity in $E$ lifting $\xi$. The field $F$ may then be identified with the field of Laurent series $\mathbb{F}_{q}((\lambda))$.

If $F_{\infty}$ is the union of the $F_{n}$, then $G=\operatorname{Gal}\left(F_{\infty} / \mathbb{Q}_{p}\right)$ acts as automorphisms on the field $F$. As with $G_{n}$, we may decompose $G=\operatorname{Gal}\left(F_{\infty} / \mathbb{Q}_{p}\right)$ into a direct product of procyclic subgroups

$$
G=\Delta \times \Gamma \times \Phi
$$

where $\operatorname{Gal}\left(F_{\infty} / E\right)=\Delta \times \Gamma$, the group $\Delta$ has order $p-1$, the group $\Gamma$ is isomorphic to $\mathbb{Z}_{p}$, and $\Phi$ is isomorphic to $\operatorname{Gal}\left(E / \mathbb{Q}_{p}\right)$. Let $\gamma$ denote the topological generator of $\Gamma$ such that $\gamma\left(\zeta_{p^{n}}\right)=\zeta_{p^{n}}^{1+p}$ for all $n$.

The pro- $p$ completion $D$ of $F^{\times}$decomposes into a direct sum of eigenspaces for the powers of the Teichmüller character $\omega$ on $\Delta$. For an integer $r$, we let $D^{(r)}=D^{\varepsilon_{r}}$, where $\varepsilon_{r}$ is the idempotent

$$
\varepsilon_{r}=\frac{1}{p-1} \sum_{\delta \in \Delta} \omega(\delta)^{-r} \delta \in \mathbb{Z}_{p}[\Delta] .
$$

For $i \geq 1$, let $U_{i}$ denote the $i$ th group in the unit filtration of $F$. We then set

$$
V_{i}^{(r)}=U_{i} \cap D^{(r)} \quad \text { and } \quad\left(V_{i}^{(r)}\right)^{\prime}=V_{i}^{(r)}-V_{i+1}^{(r)} .
$$

The following is [Sharifi 2002, Lemma 2.3] (with $F_{n}$ replaced by $F$ ).

Lemma 2.1. We have $V_{i}^{(r)} / V_{i+p-1}^{(r)} \cong \mathbb{F}_{q}$ for every $i \geq 1$, and $\left(V_{i}^{(r)}\right)^{\prime} \neq \varnothing$ if and only if $i \equiv r \bmod p-1$.

From now on, we set $V_{i}=V_{i}^{(r)}$ and $V_{i}^{\prime}=\left(V_{i}^{(r)}\right)^{\prime}$ if $i \equiv r \bmod p-1$. As a consequence of Lemma 2.1, an element $z \in V_{i}$ is determined modulo $\lambda^{i+p-1}$ by its expansion

$$
z \equiv 1+\xi \lambda^{i} \bmod \lambda^{i+1}
$$

with $\xi \in \mathbb{F}_{q}$.

The following is [Sharifi 2002, Lemma 2.4] (with $F_{n}$ replaced by $F$ ).

Lemma 2.2. Let $z \in V_{i}^{\prime}$. If $p \nmid i$, then $z^{\gamma-1} \in V_{i+p-1}^{\prime}$. Otherwise, $z^{\gamma-1} \in V_{i+2(p-1)}$. 
We identify $\Lambda=\mathbb{Z}_{p} \llbracket \Gamma \rrbracket$ with the power series ring $\mathbb{Z}_{p} \llbracket T \rrbracket$ via the continuous, $\mathbb{Z}_{p}$-linear isomorphism that takes $\gamma-1$ to $T$, and we use additive notation to describe the action of $\mathbb{Z}_{p} \llbracket T \rrbracket$ on $D$. Ramification theory would already have told us that $T \cdot V_{i} \subseteq V_{i+p-1}$ for all $i$. On the other hand, explicit calculation will yield the following two lemmas and proposition, which provide more precise information on how powers of $T$ move elements of $V_{i}$.

For $\xi \in \mathbb{F}_{q}^{\times}$, we let $V_{i}(\xi)$ denote the set of $z \in V_{i}$ for which $z$ has an expansion of the form in (2.1). We use $[k]$ to denote the smallest nonnegative integer congruent to $k \in \mathbb{Z}$ modulo $p$.

Lemma 2.3. Let $z \in V_{i}(\xi)$ for some $i$. Then, for $0 \leq j \leq[i]$, we have

$$
T^{j} z \in V_{i+j(p-1)}\left(\frac{[i] !}{([i]-j) !} \cdot \xi\right) \text {. }
$$

Proof. Note that

$$
\lambda^{\gamma}=1-\zeta^{1+p}=1-(1-\lambda)\left(1-\lambda^{p}\right)=\lambda+\lambda^{p}-\lambda^{p+1} .
$$

Using this, we see, for any $i \geq 1$, that

$$
\left(1+\xi \lambda^{i}\right)^{\gamma-1} \equiv 1+i \xi \lambda^{i+p-1} \frac{1-\lambda}{1+\xi \lambda^{i}} \bmod \lambda^{i+2 p-2} .
$$

Hence,

$$
\left(1+\xi \lambda^{i}\right)^{\gamma-1} \equiv 1+i \xi \lambda^{i+p-1} \bmod \lambda^{i+p} .
$$

Applying (2.4) recursively, we obtain the result.

Lemma 2.4. Let $z \in V_{p i-p+1}(\xi)$ for some $i \geq 2$. If $j$ is a nonnegative multiple of $p-1$, then $T^{j+1} z \in V_{p(i+j)}(\xi)$.

Proof. Let us begin by proving slightly finer versions of (2.3) in two congruence classes of exponents modulo $p$. For any $t \geq 1$, we have

$$
\begin{aligned}
\left(1+\xi \lambda^{p t}\right)^{\gamma-1} & =\frac{1+\xi \lambda^{p t}\left(1+\lambda^{p(p-1)}-\lambda^{p^{2}}\right)^{t}}{1+\xi \lambda^{p t}} \equiv 1 \bmod \lambda^{p(t+p-1)}, \\
\left(1+\xi \lambda^{p t+1}\right)^{\gamma-1} & =1+\xi \lambda^{p t+1} \frac{\sum_{m=1}^{p t+1}\left(\begin{array}{c}
p t+1 \\
m
\end{array}\right)\left(\lambda^{p-1}-\lambda^{p}\right)^{m}}{1+\xi \lambda^{p t+1}} \\
& \equiv 1+\xi\left(\lambda^{p(t+1)}-\lambda^{p(t+1)+1}\right) \bmod \left(\lambda^{p(t+p-1)+1}, \lambda^{p(2 t+1)+1}\right),
\end{aligned}
$$

the latter congruence following from the fact that $p \mid\left(\begin{array}{c}p t+1 \\ m\end{array}\right)$ for $2 \leq m<p$. Via some obvious inequalities, we conclude that

$$
\begin{aligned}
\left(1+\xi \lambda^{p t}\right)^{\gamma-1} & \equiv 1 \bmod \lambda^{p(t+2)} \\
\left(1+\xi \lambda^{p t+1}\right)^{\gamma-1} & \equiv\left(1+\xi \lambda^{p(t+1)}\right)\left(1-\xi \lambda^{p(t+1)+1}\right) \bmod \lambda^{p(t+2)} .
\end{aligned}
$$


Let $x=1+\xi \lambda^{p i-p+1}$. Recursively applying (2.5) and (2.6), we see that

$$
x^{(\gamma-1)^{k+1}} \equiv\left(1+(-1)^{k} \xi \lambda^{p(i+k)}\right)\left(1+(-1)^{k+1} \xi \lambda^{p(i+k)+1}\right) \bmod \lambda^{p(i+k+1)},
$$

for any positive integer $k$, as (2.4) implies that $U_{p(i+k)}^{\gamma-1} \subseteq U_{p(i+k+1)}$. The result now follows by application of $\varepsilon_{i}$, since

$$
z^{-1} x^{\varepsilon_{i}} \in V_{p i}, \quad T^{j+1} x^{\varepsilon_{i}} \in V_{p(i+j)}(\xi), \quad \text { and } \quad T^{j+1} V_{p i} \subset V_{p(i+j+1)-1},
$$

the latter by Lemma 2.2 .

Let us use $\{k\}$ to denote the smallest nonnegative integer congruent to $k \in \mathbb{Z}$ modulo $p-1$. For $i \geq 1$ with $p \nmid i$, we define a monotonically increasing function $\phi^{(i)}: \mathbb{Z}_{\geq 0} \rightarrow \mathbb{Z}$ by $\phi^{(i)}(0)=i$ and

$$
\phi^{(i)}(a)=p a+(i-[i])+\{[i]-a\} \quad \text { for } a \geq 1 .
$$

Proposition 2.5. Let $z \in V_{i}(\xi)$ for some $i \geq 2$ with $p \nmid i$. Then, for $j \geq 1$, we have

$$
T^{j} z \in V_{\phi^{(i)}(j)}\left(\frac{[i] !}{\{[i]-j\} !} \xi\right)
$$

Proof. Lemma 2.3 implies that

$$
T^{[i]-1} z \in V_{\phi^{(i)}([i]-1)}([i] ! \cdot \xi),
$$

and note that $\phi^{(i)}([i]-1) \equiv 1 \bmod p$. Set $k=\{[i]-j\}$. Since $j+k-[i]$ is divisible by $p-1$, Lemma 2.4 then implies that

$$
T^{j+k} z \in V_{\phi^{(i)}(j+k)}([i] ! \cdot \xi) .
$$

It follows from (2.7) that

$$
\phi^{(i)}(j+k)-i=p(j+k-[i])+(p-1)[i],
$$

and so, given (2.8), Lemma 2.2 forces $T^{l} z \in V_{\phi^{(i)}(l)}^{\prime}$ for all $l \leq j+k$. In particular, applying Lemma 2.3 with $j$ replaced by $k$ and $z$ replaced by $T^{j} z$, we see that for (2.8) to hold, $T^{j} z$ must have the stated form.

Remark 2.6. The obvious analogues of the results of this section all hold at the level of $F_{n}$ for $n \geq 2$, with $\lambda$ replaced by $\lambda_{n}=1-\zeta_{p^{n}}$. In fact, Lemmas 2.1 and 2.2 were originally proven in that setting in [Sharifi 2002]. That the other results hold breaks down to the fact that $p$ is a unit times $\lambda_{n}^{p^{n-1}(p-1)}$ in $F_{n}$, which in particular tells us that (2.2) can be replaced by $\lambda_{n}^{\gamma} \equiv \lambda_{n}+\lambda_{n}^{p}-\lambda_{n}^{p+1} \bmod \lambda_{n}^{p(p-1)+1}$. 


\section{The infinite level}

3.1. Structure of the eigenspaces. In this subsection, we fix choices of certain elements that will be used throughout the paper. From now on, we let $\xi$ denote an element of $\mathbb{F}_{q}$ with $\operatorname{Tr}_{\Phi} \xi=1$, the conjugates of which form a normal basis of $\mathbb{F}_{q}$ over $\mathbb{F}_{p}$. Let $\varphi \in \Phi$ denote the Frobenius element. Let $N_{\Phi} \in \mathbb{Z}_{p}[\Phi]$ denote the norm element. Let $\zeta=\left(\zeta_{p^{n}}\right)_{n}$ be a norm-compatible system of primitive $p^{n}$ th roots of unity as before.

Let $r$ be an integer satisfying $2 \leq r \leq p$. If $2 \leq r \leq p-2$, we simply fix an element $u_{r} \in V_{r}(\xi)$. In the case that $r=p-1$, generation of $D^{(p-1)}$ requires one additional element $\pi \in D^{(p-1)}$, a non-unit, chosen along with $u_{p-1} \in V_{p-1}(\xi)$ in the lemma which follows. The case of $r=p$ shall require more work, but we will fix elements $w \in V_{1}(-\xi)$ and $u_{p} \in V_{p}(\xi)$ as in Proposition 3.1.3 below.

Lemma 3.1.1. There exist elements $\pi \in D^{(p-1)}$ and $u_{p-1} \in V_{p-1}(\xi)$ such that $\pi^{\varphi}=\pi$ and $\pi^{\gamma-1}=u_{p-1}^{N_{\Phi}}$.

Proof. Set $\pi=\lambda^{\varepsilon_{p-1}}$, which satisfies $\pi^{\varphi}=\pi$ and $\pi^{\gamma-1} \in V_{p-1}$ (1). Since every unit is a norm in an unramified extension, there exists $u_{p-1}^{\prime} \in D^{(p-1)}$ such that $\left(u_{p-1}^{\prime}\right)^{N_{\Phi}}=\pi^{\gamma-1}$, and such an element must lie in $V_{p-1}\left(\xi^{\prime}\right)$ for some $\xi^{\prime} \in \mathbb{F}_{q}$ with $\operatorname{Tr}_{\Phi} \xi^{\prime}=1$. Hilbert's Theorem 90 tells us that $\xi^{\prime}=\xi+(\varphi-1) \eta$ for some $\eta \in \mathbb{F}_{q}$. Let $z \in V_{p-1}(\eta)$, and set $u_{p-1}=u_{p-1}^{\prime} z^{1-\varphi}$.

In fact, one could have chosen $u_{p-1} \in V_{p}(\xi)$ arbitrarily and then taken $\pi$ to satisfy the relations, as can be seen using the results of the following section.

Lemma 3.1.2. There exist elements $w \in V_{1}(-\xi)$ and $u_{p} \in V_{p}(\xi)$ with $w^{N_{\Phi}}=\zeta$ and $u_{p}^{\varphi-1}=w^{\gamma-1-p}$.

Proof. First, local class field theory yields the existence of an element $w^{\prime} \in D^{(p)}$ with $\left(w^{\prime}\right)^{N_{\Phi}}=\zeta$. Since $\zeta \in V_{1}(-1)$, we must have $w^{\prime} \in V_{1}\left(-\xi^{\prime}\right)$ for some $\xi^{\prime} \in \mathbb{F}_{q}$ with $\operatorname{Tr}_{\Phi} \xi^{\prime}=1$. Since $\xi^{\prime}=\xi+(\varphi-1) \eta$ for some $\eta \in \mathbb{F}_{q}$, we choose any $y \in V_{1}(\eta)$, and then $w=w^{\prime} y^{1-\varphi} \in V_{1}(-\xi)$ satisfies $w^{N_{\Phi}}=\zeta$ as well.

Next, note that $\left(w^{\gamma-1-p}\right)^{N_{\Phi}}=1$, and so Hilbert's Theorem 90 allows us to choose an element $u_{p}^{\prime} \in D^{(p)}$ with $\left(u_{p}^{\prime}\right)^{\varphi-1}=w^{\gamma-1-p}$. A simple computation using (2.4) tells us that $w^{\gamma-1-p} \in V_{p}\left(\xi^{p}-\xi\right)$, and therefore $u_{p}^{\prime} \in V_{p}(\xi+a)$ for some $a \in \mathbb{F}_{p}$. We may then choose $z \in V_{p}(a)$ with $z^{\varphi}=z$ and take $u_{p}=u_{p}^{\prime} z^{-1} \in V_{p}(\xi)$.

We need slightly finer information on the relationship between $w$ and $u_{p}$ inside the unit filtration, as found in the following proposition.

Proposition 3.1.3. There exist elements $w \in V_{1}(-\xi)$ and $u_{p} \in V_{p}(\xi)$ with $w^{N_{\Phi}}=\zeta$ and $u_{p}^{\varphi-1}=w^{\gamma-1-p}$ such that the element $y=u_{p} w^{p \varphi^{-1}}$ lies in $V_{2 p-1}(-\xi)$. 
Proof. For now, fix any choices of $u_{p}$ and $w$ as in Lemma 3.1.2. We must have $u_{p}=\left(1+\xi \lambda^{p}\right)^{\varepsilon_{1}} \alpha$ with $\alpha \in V_{2 p-1}$ and $w=(1-\xi \lambda)^{\varepsilon_{1}} \beta$ with $\beta \in V_{p}$. Note that

$$
\begin{aligned}
\left(1+\xi \lambda^{p}\right)^{\varphi-1} & \equiv 1+\left(\xi^{p}-\xi\right) \lambda^{p} \bmod \lambda^{2 p}, \\
(1-\xi \lambda)^{\gamma-1-p} & =\frac{1-\xi\left(\lambda+\zeta \lambda^{p}\right)}{(1-\xi \lambda)\left(1-\xi^{p} \lambda^{p}\right)} \equiv 1+\left(\xi^{p}-\xi \frac{1-\lambda}{1-\xi \lambda}\right) \lambda^{p} \bmod \lambda^{2 p} .
\end{aligned}
$$

We then have

$$
\frac{(1-\xi \lambda)^{\gamma-1-p}}{\left(1+\xi \lambda^{p}\right)^{\varphi-1}} \equiv 1+\frac{\xi(1-\xi)}{1-\xi \lambda} \lambda^{p+1} \bmod \lambda^{2 p}
$$

We denote the quantity on the right side of (3.1.1) by $\theta$. By Lemma 2.2, we have $\beta^{\gamma-1-p} \in V_{3 p-2}$, from which it follows that $\alpha^{\varphi-1} \theta^{-\varepsilon_{1}} \in V_{3 p-2}$. On the other hand, by Lemma 2.1, we have

$$
y \alpha^{-1}=\left(1+\xi \lambda^{p}\right)^{\varepsilon_{1}}\left(1-\xi \lambda^{p}\right)^{\varepsilon_{1}} \beta^{p \varphi^{-1}} \in V_{3 p-2},
$$

so in fact we have $y^{\varphi-1} \theta^{-\varepsilon_{1}} \in V_{3 p-2}$. If we can show that $\theta^{\varepsilon_{1}} \in V_{2 p-1}\left(\xi-\xi^{p}\right)$, we will then have $y \in V_{2 p-1}(-\xi+a)$ for some $a \in \mathbb{F}_{p}$. As in the proof of Lemma 3.1.2, we can then choose an element $z \in V_{2 p-1}(a)$ with $z^{\varphi}=z$ and replace $u_{p}$ by $u_{p} z^{-1}$ to obtain the result.

By Proposition 2.5, we see that to show that $\theta^{\varepsilon_{1}} \in V_{2 p-1}\left(\xi-\xi^{p}\right)$, it suffices to show that $\theta^{\varepsilon_{1}(\gamma-1)^{p-1}} \in V_{p^{2}}\left(\xi^{p}-\xi\right)$. Since $p^{2} \equiv 1 \bmod p-1$, for this, it suffices to show that

$$
\theta^{(\gamma-1)^{p-1}} \equiv 1+\left(\xi^{p}-\xi\right) \lambda^{p^{2}} \bmod \lambda^{p^{2}+1} .
$$

This is a simple consequence of Lemma 3.1.4, which follows. That is, in the notation of said lemma, Fermat's little theorem and the binomial theorem tell us that $d_{p-1, k}=-1$ for all positive integers $k \leq p-1$.

Lemma 3.1.4. For each positive integer $j \leq p-1$, one has

$$
\left(1+\frac{\xi(1-\xi)}{1-\xi \lambda} \lambda^{p+1}\right)^{(\gamma-1)^{j}} \equiv 1+\left(\sum_{k=1}^{j} d_{j, k} \xi^{k}(1-\xi)\right) \lambda^{(j+1) p} \bmod \lambda^{(j+1) p+1}
$$

where

$$
d_{j, k}=\sum_{h=1}^{k}(-1)^{j+h}\left(\begin{array}{l}
k \\
h
\end{array}\right) h^{j} \in \mathbb{F}_{p}
$$

for positive integers $k \leq j$.

Proof. We make the expansion

$$
\theta=1+\frac{\xi(1-\xi)}{1-\xi \lambda} \lambda^{p+1} \equiv \prod_{k=1}^{p-1}\left(1+\xi^{k}(1-\xi) \lambda^{p+k}\right) \bmod \lambda^{2 p}
$$


Since $U_{s}^{\gamma-1} \subseteq U_{s+p-1}$ for all $s$, as follows from (2.4), to compute $\theta^{(\gamma-1)^{j}}$ modulo $\lambda^{(j+1) p+1}$, it suffices to compute $\left(1+\xi^{k}(1-\xi) \lambda^{p+k}\right)^{(\gamma-1)^{j}}$ modulo $\lambda^{(j+1) p+1}$.

Fix a positive integer $k \leq p-1$. We claim that the coefficient of $\lambda^{(j+1) p}$ in the expansion of $\left(1+\xi^{k}(1-\xi) \lambda^{p+k}\right)^{(\gamma-1)^{j}}$ as a power series in $\mathbb{F}_{q} \llbracket \lambda \rrbracket$ is 0 if $j<k$ and $\xi^{k}(1-\xi) d_{j, k}$ if $j \geq k$. As a consequence of (2.3), one sees that

$$
\left(1+\xi \lambda^{t}\right)^{\gamma-1} \equiv\left(1+t \xi \lambda^{t+p-1}\right)\left(1-t \xi \lambda^{t+p}\right) \bmod \lambda^{t+2 p-2} \quad \text { for any } t \geq p-1 .
$$

Using this and the finer congruence (2.6) when possible, an induction yields that the expansion in question is determined by

$$
\prod_{m=0}^{\min (j, k)} \prod_{\left(a_{i}\right) \in P_{j, k, m}}\left(1+\xi^{k}(1-\xi) \frac{k !}{(k-m) !} \prod_{i=1}^{j-m} a_{i} \cdot \lambda^{(j+1) p+k-m}\right)^{(-1)^{j-m}} \bmod \lambda^{(j+1) p+k+1},
$$

where

$$
P_{j, k, m}=\left\{\left(a_{1}, a_{2}, \ldots, a_{j-m}\right) \in \mathbb{Z}^{j-m} \mid k-m \leq a_{1} \leq a_{2} \leq \cdots \leq a_{j-m} \leq k\right\}
$$

if $j>m$ and $P_{j, k, j}=\{0\}$, and we consider the empty product to be 1. In particular, the coefficient in question is indeed 0 for $j<k$ and is $\xi^{k}(1-\xi) c_{j, k}$ for $j \geq k$, where

$$
c_{j, k}=(-1)^{j-k} k ! \sum_{\left(a_{i}\right) \in P_{j, k, k}} \prod_{i=1}^{j-k} a_{i} .
$$

It remains to verify that $c_{j, k}=d_{j, k}$ for $j \geq k$.

Let $D$ denote the differential operator $x \frac{d}{d x}$ on $\mathbb{F}_{p}[x]$. By the binomial theorem, we have

$$
\left.D^{j}\left((1-x)^{k}\right)\right|_{x=1}=\left.\sum_{h=1}^{k}(-1)^{h}\left(\begin{array}{l}
k \\
h
\end{array}\right) h^{j} x^{h}\right|_{x=1}=(-1)^{j} d_{j, k} .
$$

On the other hand, repeated application of the product formula for the derivative yields

$$
\begin{aligned}
\left.D^{j}\left((1-x)^{k}\right)\right|_{x=1} & =\left.(-1)^{k} \sum_{h=1}^{\min (j, k)} \frac{k !}{(k-h) !} \sum_{\left(a_{i}\right) \in P_{j, h, h}} \prod_{i=1}^{j-h} a_{i} \cdot(x-1)^{k-h} x^{h}\right|_{x=1} \\
& =(-1)^{j} c_{j, k}
\end{aligned}
$$

for all $j \geq k$ and hence the result.

In the next section, we will obtain the following very slight refinement of what is essentially a result of [Greither 1996, Sections 2 and 3]; see also [Sharifi 2002, Corollary 2.2]. 
Theorem 3.1.5. For $r \leq p-2$, the A-module $D^{(r)}$ is freely generated by any $u_{r} \in V_{r}(\xi)$. The A-module $D^{(p-1)}$ has a presentation

$$
D^{(p-1)}=\left\langle\pi, u_{p-1} \mid \pi^{\varphi}=\pi, u_{p-1}^{N_{\Phi}}=\pi^{\gamma-1}\right\rangle,
$$

for some $u_{p-1} \in V_{p-1}(\xi)$ and $\pi \in D^{(p-1)}$. The A-module $D^{(p)}$ has a presentation

$$
D^{(p)}=\left\langle u_{p}, w \mid w^{\gamma-1-p}=u_{p}^{\varphi-1}\right\rangle
$$

for some $u_{p} \in V_{p}(\xi)$ and $w \in V_{1}(-\xi)$ such that $w^{N_{\Phi}}=\zeta$.

3.2. Special elements. Fix $r$ such that $2 \leq r \leq p$, and define $\phi: \mathbb{Z}_{\geq 0} \rightarrow \mathbb{Z}$ by $\phi(a)=\phi^{(r)}(a)$ for $a \geq 1$. Set

$$
\delta= \begin{cases}0 & \text { if } 2 \leq r \leq p-1, \\ 1 & \text { if } r=p .\end{cases}
$$

For all $a \geq 1$, we have $\phi(a)=p(a+\delta)+\{r-\delta-a\}$, so $\phi(a)$ is the smallest integer that is at least $p(a+\delta)$ and congruent to $r$ modulo $p-1$.

From now on, $i$ will be used solely to denote a positive integer congruent to $r$ modulo $p-1$. We will write $\alpha \sim \beta$ to denote that both $\alpha$ and $\beta$ lie in $V_{i}(\xi)$ for some $i$ and $\xi \in \mathbb{F}_{q}^{\times}$. We use additive notation for the action of $A=\mathbb{Z}_{p}[\Phi] \llbracket T \rrbracket$ on $D^{(r)}$. We begin with the following useful lemma.

Lemma 3.2.1. Let $j$ be a positive integer.

a. We have

$$
T^{j} u_{r} \in V_{\phi(j)}\left(\frac{[r] !}{\{r-\delta-j\} !} \xi\right) .
$$

b. If $j \equiv r-\delta \bmod (p-1)$ so that $T^{j} u_{r} \sim$ pzfor some $z \in D^{(r)}$, then

$$
T^{j} u_{r}-p z \in V_{\phi(j)+p-1}(-[r] ! \xi) .
$$

Proof. For $r<p$, part a is a direct consequence of Proposition 2.5 and the fact that $u_{r} \in V_{r}(\xi)$. For $r=p$, Proposition 2.5 and the fact that $\phi=\phi^{(2 p-1)}$ on positive integers would tell us more directly that $T^{j} y \in V_{\phi(j)}\left(\frac{1}{\{-j\} !} \xi\right)$ for $j \geq 1$ and $y$ as in Proposition 3.1.3. Note, however, that $T u_{p}=T y-p \varphi^{-1} T w \sim T y$, since $p T w \in V_{p^{2}}$. This is also the key point of part b. That is, we have

$$
T\left(T^{j} u_{r}-p z\right) \sim T^{j+1} u_{r}
$$

as $p T z \in V_{p \phi(j)}$ and

$$
\phi(j+1) \leq \phi(j)+2(p-1)<p \phi(j) .
$$

Since $T^{j+1} u_{r} \in V_{\phi(j)+2(p-1)}([r] ! \xi)$, a final application of Proposition 2.5 tells us that $T^{j} u_{r}-p z$ had to be in the stated group. 
For a nonnegative integer $m$, let us define $\phi_{m}: \mathbb{Z}_{\geq 0} \rightarrow \mathbb{Z}_{\geq 0}$ by $\phi_{m}=p^{m}(\phi+1)-1$. We remark that

$$
p \phi_{m}=\phi \circ\left(\phi_{m}-\delta\right) .
$$

From now on, we set $\rho=p \varphi^{-1}$ for brevity of notation. We define special elements in the unit filtration of $D^{(r)}$.

Theorem 3.2.2. Let $m$ and $j$ be nonnegative integers. Define

$$
\alpha_{m, j}=\frac{1}{[r] !}\left(\{r-\delta-j\} ! \rho^{m} T^{j}-\sum_{k=1}^{m} \rho^{m-k} T^{\phi_{k-1}(j)-\delta}\right) u_{r},
$$

unless $j=0$ and $r=p-1$, in which case we replace $\{r-\delta-j\}$ ! with -1 in the formula. Then $\alpha_{m, j} \in V_{\phi_{m}(j)}(\xi)$. Furthermore, $\left(p^{m} b T^{j}+c\right) u_{r} \notin V_{\phi_{m}(j)+p-1}$ for all $b \in \mathbb{Z}_{p}[\Phi]$ with $b \not \equiv 0 \bmod p$ and $c \in T^{j+1} A$.

Proof. We work by induction, the case of $m=0$ being Lemma 3.2.1a, aside from the case $j=0$, in which case it is simply the definition of $u_{r}$. Assume we have proven the first statement for $m$. Then $p \alpha_{m, j} \in V_{p \phi_{m}(j)}\left(\xi^{p}\right)$ and, using Lemma 3.2.1a and (3.2.1), we have

$$
T^{\phi_{m}(j)-\delta} u_{r} \in V_{p \phi_{m}(j)}([r] ! \xi) .
$$

Lemma 3.2.1b then tells us that

$$
\alpha_{m+1, j}=\rho \alpha_{m, j}-\frac{1}{[r] !} T^{\phi_{m}(j)-\delta} u_{r} \in V_{p \phi_{m}(j)+p-1}(\xi) .
$$

Now assume the second statement is true for $m$. (For $m=0$, this is a consequence of the fact that the conjugates of $\xi$ are $\mathbb{F}_{p}$-linearly independent.) Suppose that

$$
\alpha=\left(p^{m+1} b T^{j}+c\right) u_{r} \in V_{i}^{\prime}
$$

with $i \geq \phi_{m+1}(j), b \in \mathbb{Z}_{p}[\Phi]-p \mathbb{Z}_{p}[\Phi]$ and $c \in T^{j+1} A$. We write $c=\left(p c^{\prime}+T^{h} v\right) u_{r}$ for some $c^{\prime}, v \in A$ with $v \not \equiv 0 \bmod (p, T)$ and $h \geq j+1$. By induction, we have

$$
\left(p^{m} b T^{j}+c^{\prime}\right) u_{r} \notin V_{\phi_{m}(j)+p-1} .
$$

Since $\phi_{m+1}(j)=p \phi_{m}(j)+p-1$ and $\alpha \in V_{\phi_{m+1}(j)}$ by assumption, this forces

$$
p\left(p^{m} b T^{j}+c^{\prime}\right) u_{r} \sim-T^{h} v u_{r},
$$

which tells us by Lemma 3.2.1a that $\phi(h) \leq p \phi_{m}(j)$. On the other hand, it follows from Lemma 3.2.1b that $\alpha \in V_{\phi(h)+p-1}^{\prime}$, which forces $i=\phi_{m+1}(j)$.

The second statement of Theorem 3.2.2 insures, in particular, that $a u_{r} \neq 0$ for all nonzero $a \in A$. We therefore have the following corollary.

Corollary 3.2.3. The A-submodule of $D^{(r)}$ generated by $u_{r}$ is free. 
In the exceptional case that $r=p$, we require additional elements. First, we modify the function $\phi_{m}$ for this $r$. For nonnegative integers $m$ and $j$, we set $\phi_{m}^{\prime}(j)=\phi_{m}(j)$ unless $r=p$ and $j=p^{l}-1$ for some $l \geq 0$, in which case we set

$$
\phi_{m}^{\prime}\left(p^{l}-1\right)=p^{m+l+1}+p^{m+1}-1=\phi_{m}\left(p^{l}-1\right)+p^{m}(p-1) .
$$

Theorem 3.2.4. Let $l$ and $m$ be nonnegative integers. Define

$$
\beta_{m, l}=\left(\rho^{m} T^{p^{l}-1}+\sum_{k=1}^{m} \rho^{m-k} T^{\phi_{k-1}^{\prime}\left(p^{l}-1\right)-1}\right) u_{p}+\rho^{m+l+1} w .
$$

Then $\beta_{m, l} \in V_{\phi_{m}^{\prime}\left(p^{l}-1\right)}(-\xi)$. Moreover, for any $j \geq 0$, we have

$$
\left(p^{m} b T^{j}+c\right) u_{p}+d w \notin V_{\phi_{m}^{\prime}(j)+p-1}
$$

for all $b \in \mathbb{Z}_{p}[\Phi]-p \mathbb{Z}_{p}[\Phi], c \in T^{j+1} A$ and $d \in \mathbb{Z}_{p}[\Phi]$.

Proof. The proof is similar to that of Theorem 3.2.2. Since Lemma 3.2.1a and the definition of $w$ tell us that

$$
T^{p^{l}-1} u_{p} \in V_{p^{l+1}}(\xi) \quad \text { and } \quad \rho^{l+1} w \in V_{p^{l+1}}(-\xi),
$$

Lemma 3.2.1b yields $\beta_{0, l}=\rho^{l+1} w+T^{p^{l}-1} u_{p} \in V_{p^{l+1}+p-1}(-\xi)$. For any $m \geq 0$, we have

$$
\beta_{m+1, l}=\rho \beta_{m, l}+T^{\phi_{m}^{\prime}\left(p^{l}-1\right)-1} u_{p} .
$$

By induction and Lemma 3.2.1a, we have

$$
\rho \beta_{m, l} \in V_{p \phi_{m}^{\prime}\left(p^{l}-1\right)}(-\xi) \quad \text { and } \quad T^{\phi_{m}^{\prime}\left(p^{l}-1\right)-1} u_{p} \in V_{p \phi_{m}^{\prime}\left(p^{l}-1\right)}(\xi) .
$$

Since $\phi_{m+1}^{\prime}\left(p^{l}-1\right)=p \phi_{m}^{\prime}\left(p^{l}-1\right)+p-1$, that $\beta_{m+1, l} \in V_{\phi_{m+1}^{\prime}\left(p^{l}-1\right)}(-\xi)$ is just another application of Lemma 3.2.1b.

Let $j \geq 0, b \in \mathbb{Z}_{p}[\Phi]-p \mathbb{Z}_{p}[\Phi], c \in T^{j+1} A$, and $d \in \mathbb{Z}_{p}[\Phi]$. First, suppose that $\alpha=\left(b T^{j}+c\right) u_{p}+d w \in V_{i}^{\prime}$ for some $i \geq \phi_{0}^{\prime}(j)$. Note that $\left(b T^{j}+c\right) u_{p} \in V_{\phi(j)}^{\prime}$ by Lemma 3.2.1a, while $d w \in V_{p^{l}}^{\prime}$ for some $l \geq 0$. Since $\alpha \in V_{\phi_{0}^{\prime}(j)}$, we must have $p^{l} \geq \phi(j)$. We then have $\alpha \in V_{\phi(j)}^{\prime}$ unless $\phi(j)=p^{l}$. This occurs if and only if $l \geq 1$ and $j=p^{l-1}-1$, in which case $\phi_{0}^{\prime}(j)=p^{l}+p-1$. For this to hold, we must have $\left(b T^{j}+c\right) u_{p} \sim-d w$. Lemma 3.2.1b then implies that $\alpha \in V_{p^{l}+p-1}^{\prime}$, so $i=\phi_{0}^{\prime}(j)$ in all cases.

Suppose now that $\alpha=\left(p^{m+1} b T^{j}+c\right) u_{p}+d w \in V_{i}^{\prime}$ for some $i \geq \phi_{m+1}^{\prime}(j)$. Rewrite $c$ as $p c^{\prime}+T^{h} v$ for some $h \geq j+1$ and $c^{\prime}, v \in A$ with $v \not \equiv 0 \bmod (p, T)$. If we are to have $\alpha \in V_{p}$, we may also write $d=p d^{\prime}$ for some $d^{\prime} \in \mathbb{Z}_{p}[\Phi]$. By induction, we have

$$
\left(p^{m} b T^{j}+c^{\prime}\right) u_{p}+d^{\prime} w \notin V_{\phi_{m}^{\prime}(j)+p-1},
$$


and so in order that $\alpha \in V_{\phi_{m+1}^{\prime}}(j)$, we must have

$$
\left(p^{m+1} b T^{j}+p c^{\prime}\right) u_{p}+d w \sim-T^{h} v u_{p},
$$

which tells us using Lemma 3.2.1a that $\phi(h) \leq p \phi_{m}^{\prime}(j)$. On the other hand, Lemma 3.2.1b tells us that $\alpha \in V_{\phi(h)+p-1}^{\prime}$, so we must have $i=\phi_{m+1}^{\prime}(j)$.

Theorem 3.1.5 may now be proven as a consequence of the description of the elements above and their place in the unit filtration.

Proof of Theorem 3.1.5. For $r \leq p-1$, the union of the disjoint images of the functions $\phi_{m}$ is exactly the set of positive integers congruent to $r$ modulo $p-1$. Therefore, Theorem 3.2.2 implies that there exists an element of the $A$-module generated by $u_{r}$ in $V_{i}(\xi)$ for each $i \equiv r \bmod p-1$. In particular, $u_{r}$ therefore clearly generates $V_{r}$ as an $A$-module, which equals $D^{(r)}$ for $r \leq p-2$, and it is free by Corollary 3.2.3. Every element of $D^{(p-1)}$ may then be written in the form $\pi^{m} u_{p-1}^{a}$ with $m \in \mathbb{Z}_{p}$ and $a \in A$, and such an element can clearly only be trivial if $m$ is, and therefore $a$ is as well. Noting that our choices of $\pi$ and $u_{p-1}$ as in Lemma 3.1.1 satisfy the desired relations, the presentation for $r=p-1$ is as stated.

For $r=p$, the union of $\{1\}$ and the images of the functions $\phi_{m}$ and $\phi_{m}^{\prime}$ is the set of positive integers that are congruent to 1 modulo $p-1$. Theorem 3.2.2 and Theorem 3.2.4 imply that there exists an element of the $A$-module generated by $u_{p}$ and $w$ in $V_{i}(\xi)$ for each $i \equiv 1 \bmod p-1$. Thus, this $A$-module is $D^{(p)}$. Our choices of $u_{p}$ and $w$ satisfy the relations of Lemma 3.1.2, and it follows from the second statement of Theorem 3.2.4 that if either $c \in A$ or $d \in \mathbb{Z}_{p}[\Phi]$ is nonzero, then so is $c u_{p}+d w$.

3.3. Refined elements. In this section, we provide refinements of the elements constructed in Theorem 3.2.2 and Theorem 3.2.4. We maintain the notation of Section 3.2. We begin by constructing certain one-sided inverses to the monotonically increasing functions $\phi$ and $\phi_{m}$.

For any nonnegative integer $a$ and positive integer $t$, let us set

$$
\langle a\rangle_{t}=\max (a+\{t-a\}, t) .
$$

Therefore, $\langle a\rangle_{t}$ is the smallest integer greater than or equal to $t$ and $a$ and congruent to $t$ modulo $p-1$. Define $\psi: \mathbb{Z}_{\geq 0} \rightarrow \mathbb{Z}_{\geq 0}$ by

$$
\psi(a)=\left\lfloor\frac{\langle a\rangle_{r}+1}{p}\right\rfloor-\delta
$$

except for $r=p-1$ and $a \leq p-1$, in which case we set $\psi(a)=0$. For $m \geq 0$, define $\psi_{m}: \mathbb{Z}_{\geq 0} \rightarrow \mathbb{Z}_{\geq 0}$ by

$$
\psi_{m}(a)=\psi\left(\left\lceil\frac{a+1}{p^{m}}\right\rceil-1\right) .
$$


Note that $\psi_{0}=\psi$.

Lemma 3.3.1. We have $\psi_{m}\left(\phi_{m}(j)\right)=j$ for all nonnegative integers $j$. Moreover, for all such $j$ and positive integers $a$, we have $\phi_{m}(j) \geq a$ if and only if $j \geq \psi_{m}(a)$. Proof. First, note that $\phi(j)$ is congruent to $r$ modulo $p-1$, so we have

$$
\psi(\phi(j))=\left\lfloor\frac{\phi(j)+1}{p}\right\rfloor-\delta=\left\lfloor\frac{p(j+\delta)+\{r-\delta-j\}+1}{p}\right\rfloor-\delta=j,
$$

unless $r \geq p-1$ and $j=0$, but one checks immediately that $\psi(\phi(0))=\psi(r)=0$ if $r \geq p-1$ as well. It follows that we have

$$
\psi_{m}\left(\phi_{m}(j)\right)=\psi\left(\left\lceil\frac{p^{m}(\phi(j)+1)}{p^{m}}\right\rceil-1\right)=\psi(\phi(j))=j .
$$

Therefore, if $\phi_{m}(j) \geq a$, then $j=\psi_{m}\left(\phi_{m}(j)\right) \geq \psi_{m}(a)$, since $\psi_{m}$ is nondecreasing.

To finish the proof, we need only show that $\phi_{m}\left(\psi_{m}(a)\right) \geq a$, since $\phi_{m}$ is nondecreasing (in fact, strictly increasing). First, note that the definition of $\psi$ is such that $\psi(a)=\psi\left(\langle a\rangle_{r}\right)$. For $i \equiv r \bmod p-1$ with $i \neq 1, p-1$, the value $\phi(\psi(i))$ is the unique integer between $p\lfloor(i+1) / p\rfloor$ and $p\lfloor(i+1) / p\rfloor+p-2$ that is congruent to $r \bmod p-1$. This implies that

$$
\phi(\psi(a))= \begin{cases}\langle a\rangle_{r} & \text { if }\langle a\rangle_{r} \not \equiv-1 \bmod p, \text { or } a \leq r=p-1, \\ \langle a\rangle_{r}+p-1 & \text { otherwise, }\end{cases}
$$

which is, in particular, at least $a$. By definition of $\phi_{m}$ and $\psi_{m}$, we then have

$$
\phi_{m}\left(\psi_{m}(a)\right)=p^{m}\left(\phi\left(\psi_{m}(a)\right)+1\right)-1 \geq p^{m}\left\lceil\frac{a+1}{p^{m}}\right\rceil-1 \geq a .
$$

We actually need a version of Lemma 3.3.1 with $\phi_{m}$ replaced by $\phi_{m}^{\prime}$ and $\psi_{m}$ replaced by an appropriate function $\psi_{m}^{\prime}: \mathbb{Z}_{\geq 0} \rightarrow \mathbb{Z}_{\geq 0}$, which we now define. Set $\psi_{m}^{\prime}=\psi_{m}$ if $r \leq p-1$ and, if $r=p$, let

$\psi_{m}^{\prime}(a)= \begin{cases}\psi_{m}(a)-1 & \text { if } p^{m+l+1}+p^{m} \leq a \leq p^{m+l+1}+p^{m+1}-1 \text { for some } l \geq 0, \\ \psi_{m}(a) & \text { otherwise. }\end{cases}$

Note that $\psi_{m}^{\prime}(a)=\psi_{m}(a)-1$ for $r=p$ if and only if $\phi_{m}\left(p^{l}-1\right)<a \leq \phi_{m}^{\prime}\left(p^{l}-1\right)$ for some $l \geq 0$, in which case $\psi_{m}^{\prime}(a)=p^{l}-1$. One then easily checks the following:

Corollary 3.3.2. We have $\psi_{m}^{\prime}\left(\phi_{m}^{\prime}(j)\right)=j$ for all nonnegative integers $j$. Moreover, for all such $j$ and positive integers $a$, we have $\phi_{m}^{\prime}(j) \geq a$ if and only if $j \geq \psi_{m}^{\prime}(a)$.

For the rest of this section, we fix a positive integer $i$ with $i \equiv r \bmod p-1$.

Remark 3.3.3. Lemma 3.3.1 and Theorem 3.2.2 tell us that each $\alpha_{m, \psi_{m}(i)}$ lies in $V_{i}$. Corollary 3.3.2 and Theorem 3.2.4 tell us that each $\beta_{m, l}$ with $\psi_{m}^{\prime}(i)=p^{l}-1$ lies in $V_{i}$. These elements have the form $\left(p^{m} b T^{j}+c\right) u_{r}+d w$ for $j=\psi_{m}^{\prime}(i)$, where 
$b \in \mathbb{Z}_{p}[\Phi]-p \mathbb{Z}_{p}[\Phi], c \in T^{j+1} A$, and $d \in \mathbb{Z}[\Phi]$, with $d=0$ if $r \neq p$. The same results also show that no such element with $j<\psi_{m}^{\prime}(i)$ can lie in $V_{i}$.

For any $m \geq 0$, define $\theta_{m}: \mathbb{Z}_{\geq 1} \rightarrow \mathbb{Z}_{\geq 0}$ by

$$
\theta_{m}(a)=\psi\left(\left\lceil\frac{\langle a\rangle_{r}}{p^{m}}\right\rceil\right) .
$$

By Lemma 3.2.1a and Lemma 3.3.1, the value $\theta_{m}(i)$ for $i \equiv r \bmod p-1$ is the minimal integer $j$ such that $p^{m} T^{j} u_{r} \in V_{i}$. In particular, $\theta_{m}(i) \geq \psi_{m}(i)$ for all $i$.

Lemma 3.3.4. For all positive integers $m$ and $k$ with $k \leq m$, we have

$$
\phi_{k-1}^{\prime}\left(\psi_{m}^{\prime}(i)\right)-\delta \geq \theta_{m-k}(i)-1,
$$

with equality if and only if

$$
p^{m-k+1} \phi_{k-1}^{\prime}\left(\psi_{m}^{\prime}(i)\right)<i .
$$

Moreover, we have $\psi_{m}^{\prime}(i) \geq \theta_{m}(i)-1$, with equality if and only if the equivalent conditions above hold for $k=1$.

Proof. Let us check the case that $r=p$ and $\psi_{m}^{\prime}(i)=p^{l}-1$ for some $l \geq 0$ separately. First, suppose that $p^{m+l+1}<i<p^{m+l+1}+p^{m+1}$. In this case, we have $\psi_{m}^{\prime}(i)=\theta_{m}(i)-1$. We also have

$$
\phi_{k-1}^{\prime}\left(\psi_{m}^{\prime}(i)\right)=\phi_{k-1}^{\prime}\left(p^{l}-1\right)=p^{k+l}+p^{k}-1=\psi\left(p^{k+l+1}+p^{k+1}\right) \geq \theta_{m-k}(i),
$$

with equality if and only if

$$
p^{m-k+1} \phi_{k-1}^{\prime}\left(\psi_{m}^{\prime}(i)\right)=p^{m+l+1}+p^{m+1}-p^{m-k+1}<i .
$$

Moreover, in the case that $p^{m+l+1}-p^{m+1}+2 p^{m}<i \leq p^{m+l+1}$, the values $\phi_{k-1}^{\prime}\left(\psi_{m}^{\prime}(i)\right)$ and $p^{m-k+1} \phi_{k-1}^{\prime}\left(\psi_{m}^{\prime}(i)\right)$ are the same as in the previous case, while $\theta_{m-k}(i)-1$ and $i$ are smaller. So, we may assume from this point forward that $r$ and $i$ are such that $\psi_{m}^{\prime}(i)=\psi_{m}(i)$ and $\phi_{k-1}^{\prime}\left(\psi_{m}^{\prime}(i)\right)=\phi_{k-1}\left(\psi_{m}(i)\right)$ for all $k$.

We claim that $\rho^{m-k} T^{\phi_{k-1}\left(\psi_{m}(i)\right)+1-\delta} u_{r}$ lies in $V_{i+p-1}$ for all positive $k \leq m$ and that $\rho^{m-k} T \alpha_{k, \psi_{m}(i)}$ lies in $V_{i+p-1}$ for all nonnegative $k \leq m$. Note that $T \alpha_{m, \psi_{m}(i)}$ lies in $V_{i+p-1}$ as a consequence of Theorem 3.2.2. Suppose that $\rho^{m-k} T \alpha_{k, \psi_{m}(i)} \in V_{i+p-1}$ for some positive $k \leq m$. We then have

$$
\rho^{m-k} T^{\phi_{k-1}\left(\psi_{m}(i)\right)+1-\delta} u_{r} \sim-[r] ! \rho^{m-k} T \alpha_{k, \psi_{m}(i)} \in V_{i+p-1},
$$

which also forces $\rho^{m-k+1} T \alpha_{k-1, \psi_{m}(i)} \in V_{i+p-1}$, since

$$
\rho^{m-k+1} T \alpha_{k-1, \psi_{m}(i)}=\rho^{m-k} T \alpha_{k, \psi_{m}(i)}+\frac{1}{[r] !} \rho^{m-k} T^{\phi_{k-1}\left(\psi_{m}(i)\right)+1-\delta} u_{r},
$$


proving the claim. In particular, since $\rho^{m} T \alpha_{0, \psi_{m}(i)} \in V_{i+p-1}$, we have

$$
\rho^{m} T^{\psi_{m}(i)+1} u_{r} \in V_{i+p-1}
$$

as well. The definition of $\theta_{m-k}(i)$ now yields the desired inequalities.

Now (3.3.2) holds for a given $k$ if and only if $\rho^{m-k+1} \alpha_{k-1, \psi_{m}(i)} \notin V_{i}$. Since

$$
[r] ! \rho \alpha_{k-1, \psi_{m}(i)} \sim T^{\phi_{k-1}\left(\psi_{m}(i)\right)-\delta} u_{r},
$$

this occurs if and only if $\rho^{m-k} T^{\phi_{k-1}\left(\psi_{m}(i)\right)-\delta} u_{r} \notin V_{i}$ and, therefore, if and only if

$$
\phi_{k-1}\left(\psi_{m}(i)\right)-\delta \leq \theta_{m-k}(i)-1 \text {, }
$$

which must then be an equality. Also, $\psi_{m}(i)<\theta_{m}(i)$ if and only if $\rho^{m} \alpha_{0, \psi_{m}(i)} \notin V_{i}$, which holds by Lemma 3.2.1a if and only if $p^{m} \phi\left(\psi_{m}(i)\right)<i$, the same condition as (3.3.2) for $k=1$.

From now on, we set $i_{m}=\left\lceil\frac{i}{p^{m}}\right\rceil$ for all $m \geq 0$.

Lemma 3.3.5. For any pair of positive integers $m$ and $k$ with $k \leq m$, we have $\phi_{k-1}^{\prime}\left(\psi_{m}^{\prime}(i)\right)-\delta \geq \theta_{m-k}(i)-1$, with equality if and only if

(1) $i_{m+\epsilon} \not \equiv 0 \bmod p$, or $r=p-1$ and $i_{m}=p$,

(2) $i_{m+\epsilon} \equiv r+1 \bmod p-1$, but not $r=p-1$ and $i_{m}=1$, and

(3) $i \equiv-j \bmod p^{m+\epsilon}$ for some $0<j<p^{m+1-k}$,

where $\epsilon=0$ unless $r=p$ and $i_{m+1}=p^{l}+1$ for some $l \geq 0$, in which case we set $\epsilon=1$. Moreover, we have $\psi_{m}^{\prime}(i) \geq \theta_{m}(i)-1$, with equality if and only if the above conditions hold with $k=1$.

Proof. The case that $r=p$ and $\psi_{m}^{\prime}(i)=p^{l}-1$ for some $l \geq 0$ follows from the proof of Lemma 3.3.4, noting that if $i_{m+1}=p^{l}+1$, then it is both nonzero modulo $p$ and congruent to $p+1$ modulo $p-1$, and the third condition of the lemma holds exactly when (3.3.3) does. On the other hand, for the remaining $i$ with $\psi_{m}^{\prime}(i)=p^{l}-1$, we have $i_{m+1}=p^{l}$, and the fact that the inequality is strict was shown in the proof of Lemma 3.3.4. So, we again assume that $r \neq p$ or $i$ is such that $\psi_{m}^{\prime}(i) \neq p^{l}-1$ for all $l \geq 0$.

By Lemma 3.3.4, it suffices to determine the precise conditions under which (3.3.2) holds. Let us set $a=(i+1)_{m}$. It follows from (3.3.1) that we have

$p^{m-k+1} \phi_{k-1}\left(\psi_{m}(i)\right)= \begin{cases}p^{m}\langle a\rangle_{r+1}-p^{m-k+1} & \text { if } p \nmid\langle a\rangle_{r+1}, \\ p^{m}\langle a\rangle_{r+1}+p^{m}(p-1)-p^{m-k+1} & \text { otherwise, }\end{cases}$

unless $r=p-1$ and $\langle a\rangle_{r+1}=p$, in which case

$$
p^{m-k+1} \phi_{k-1}\left(\psi_{m}(i)\right)=p^{m+1}-p^{m-k+1} .
$$


Aside from this exceptional case, (3.3.4) implies that $p$ cannot divide $\langle a\rangle_{r+1}$ if (3.3.2) is to hold. Moreover, if $\langle a\rangle_{r+1}>a$, then again (3.3.2) cannot hold, so for it to hold, we must have $a \equiv r+1 \bmod p-1$, but not $r=p-1$ and $a=1$. Assuming that these necessary conditions hold, the condition that

$$
p^{m-k+1} \phi_{k-1}\left(\psi_{m}(i)\right)=p^{m} a-p^{m-k+1}<i
$$

is exactly that $i \equiv-j \bmod p^{m}$ with $0<j<p^{m-k+1}$.

For $m \geq 0$, we will define new elements $\kappa_{m, i}$ of $V_{i}$ that involve fewer terms and easier-to-compute exponents of powers of $T$ than the expressions for $\alpha_{m, \psi_{m}(i)}$ and $\beta_{m, l}$. In preparation, set $\sigma(m, i)=\left\lfloor\log _{p}\left(p^{m} i_{m}-i\right)\right\rfloor$ for any $m \geq 0$ such that $p^{m} \nmid i$. Note that $0 \leq \sigma(m, i) \leq m-1$ when it is defined and $\sigma(m+1, i)$ is defined and greater than or equal to $\sigma(m, i)$ whenever $\sigma(m, i)$ is defined.

First, supposing either that $r \leq p-1$ or that $r=p$ and $i_{m+1}-1$ is not a power of $p$, we set

$$
\kappa_{m, i}=\rho^{m} T^{\theta_{m}(i)} u_{r}
$$

if $i_{m} \not \equiv r+1 \bmod p-1, p \mid i_{m}, i<p^{m}$, or $p^{m} \mid i$, unless $r=p-1$ and $i_{m}=p$, and

$$
\kappa_{m, i}=\left(\rho^{m} T^{\theta_{m}(i)-1}-a_{m, i} \sum_{k=\sigma(m, i)}^{m-1} \rho^{k} T^{\theta_{k}(i)-1}\right) u_{r}
$$

otherwise, where $a_{m, i}$ denotes the least positive residue of $\left(\left\{r+1-\delta-\theta_{m}(i)\right\} !\right)^{-1}$ modulo $p$ unless $r=p-1$ and $\theta_{m}(i)=1$, in which case we take $a_{m, i}=-1$. In the remaining case that $r=p$ and $i_{m+1}-1$ is a power of $p$, we set

$$
\kappa_{m, i}=\left(\rho^{m} T^{\theta_{m}(i)-1}+\sum_{k=\sigma(m+1, i)}^{m-1} \rho^{k} T^{\theta_{k}(i)-1}\right) u_{r}+\rho^{m+\log _{p}\left(i_{m+1}-1\right)+1} w .
$$

For consistency, we let $a_{m, i}=-1$ for such $m$. Note that Lemma 3.3.5 tells us that each $\kappa_{m, i}$ has the form $\left(\rho^{m} T^{\psi_{m}^{\prime}(i)}+c\right) u_{r}+d w$ for some $c \in T^{\psi_{m}^{\prime}(i)+1} A$ and $d \in \mathbb{Z}_{p}[\Phi]$, with $d$ taken to be zero if $r \leq p-1$.

We give two examples for $p=5$ and particular values of $i$.

Example 3.3.6. Suppose that $p=5, r=3$, and $i=11899$. Then we have

$$
\begin{aligned}
\kappa_{0, i} & =T^{2380} u_{3}, & \kappa_{1, i} & =\rho T^{476} u_{3}, \\
\kappa_{2, i} & =\left(\rho^{2} T^{95}-\rho T^{475}-T^{2379}\right) u_{3}, & \kappa_{3, i} & =\left(\rho^{3} T^{19}-\rho^{2} T^{95}\right) u_{3}, \\
\kappa_{4, i} & =\rho^{4} T^{4} u_{3}, & \kappa_{5, i} & =\left(\rho^{5}-\rho^{4} T^{3}-\rho^{3} T^{19}\right) u_{3} .
\end{aligned}
$$


Example 3.3.7. Suppose that $p=5, r=5$, and $i=92729$. Then we have

$$
\begin{array}{rlrl}
\kappa_{0, i} & =T^{18545} u_{5}, & & \kappa_{1, i}=\left(\rho T^{3708}-T^{18544}\right) u_{5}, \\
\kappa_{2, i}=\rho^{2} T^{741} u_{5}, & \kappa_{3, i}=\left(\rho^{3} T^{147}-\rho^{2} T^{740}-\rho T^{3708}\right) u_{5}, \\
\kappa_{4, i}=\rho^{4} T^{29} u_{5}, & \kappa_{5, i}=\left(\rho^{5} T^{4}+\rho^{4} T^{28}\right) u_{5}+\rho^{7} w, \\
\kappa_{6, i}=\rho^{6} u_{5}+\rho^{7} w . &
\end{array}
$$

Remark 3.3.8. It is not hard to see from the definition of $\sigma(m, i)$ that $\sigma(m, i) \geq k$ for $k<m$ if and only if $p^{m-k} \nmid i_{k}$. Moreover, if for a given $k$ there exists $m>k$ such that $\sigma(m, i)$ is less than $k$ or not defined, then $p \mid i_{k}$ so $\kappa_{k, i}=\rho^{k} T^{\theta_{k}(i)} u_{r}$ unless $r=p$ and $i_{k+1}-1$ is a power of $p$ or $r=p-1$ and $i_{k}=p$. The previous examples illustrate some of this.

Let us show that the $\kappa_{m, i}$ are actually elements of $V_{i}$. In the process, we see how they compare to the elements $\alpha_{m, \psi_{m}(i)}$ and $\beta_{m, l}$ previously defined.

Proposition 3.3.9. The elements $\kappa_{m, i}$ lie in $V_{i}$ for all nonnegative integers $m$.

Proof. Suppose first that $r \neq p$ or $i$ does not satisfy $i_{m}=p^{l}+1$ for any $l \geq 0$ (and omitting the case $r=p-1$ and $\psi_{m}(i)=0$, for which one should take the fractions in the following two equations to be 1$)$. If $\psi_{m}(i)=\theta_{m}(i)$, then we have

$$
\kappa_{m, i}=\frac{[r] !}{\left\{r-\delta-\psi_{m}(i)\right\} !} \rho^{m} \alpha_{0, \psi_{m}(i)},
$$

and this lies in $V_{i}$ by the definition of $\theta_{m}(i)$. If $\psi_{m}(i)=\theta_{m}(i)-1$, we claim that

$$
\kappa_{m, i} \sim \frac{[r] !}{\left\{r-\delta-\psi_{m}(i)\right\} !} \rho^{\sigma(m, i)} \alpha_{m-\sigma(m, i), \psi_{m}(i)} .
$$

To see this, note that

$$
\kappa_{m, i}=\rho^{\sigma(m, i)}\left(\rho^{m-\sigma(m, i)} T^{\psi_{m}(i)}-a_{m, i} \sum_{k=1}^{m-\sigma(m, i)} \rho^{m-\sigma(m, i)-k} T^{\theta_{m-k}(i)-1}\right) u_{r} .
$$

It follows from Lemma 3.3.5 that $\theta_{m-k}(i)-1=\phi_{k-1}\left(\psi_{m}(i)\right)-\delta$ if and only if $p^{m-k+1}>p^{m} i_{m}-i$, and therefore if $k \leq m-\sigma(m, i)$, proving the claim. (Note that we the reason we do not have actual equality in (3.3.8) is simply that we took $a_{m, i}$ to be an inverse to $\left\{r-\delta-\psi_{m}(i)\right\}$ ! modulo $p$, not in $\mathbb{Z}_{p}^{\times}$.) Moreover, we have by Theorem 3.2.2 that $\kappa_{m, i} \in V_{t}$ with $t=p^{\sigma(m, i)} \phi_{m-\sigma(m, i)}\left(\psi_{m}(i)\right)$. Since $p^{\sigma(m, i)} \leq p^{m} i_{m}-i$, Lemma 3.3.5 implies that

$$
\phi_{m-\sigma(m, i)}\left(\psi_{m}(i)\right)-\delta \geq \theta_{\sigma(m, i)-1}(i),
$$

and Lemma 3.3.4 then states that $t \geq i$. 
Finally, if $r=p$ and $i_{m+1}=p^{l}+1$ for some $l \geq 0$, then Lemma 3.3.5 similarly implies that $\kappa_{m, i}=\rho^{\sigma(m+1, i)} \beta_{m-\sigma(m+1, i), l}$. By Theorem 3.2.4, we have in this case that $\kappa_{m, i} \in V_{t}$ with

$$
t=p^{\sigma(m+1, i)} \phi_{m-\sigma(m+1, i)}^{\prime}\left(p^{l}-1\right) \geq i,
$$

the inequality again following from Lemmas 3.3.4 and 3.3.5.

3.4. Generating sets. In this subsection, we give explicit minimal generating sets of all of the $A$-modules $V_{i}$ in terms of the elements $\kappa_{m, i}$ of the previous section. We begin with generation. Recall that $\delta \in\{0,1\}$ is 1 if and only if $r=p$.

Theorem 3.4.1. We let $S_{i}=\left\{\kappa_{m, i} \mid 0 \leq m \leq s\right\}$ for

$$
s=\left\lceil\log _{p}\left(\frac{i+1}{r+1+\delta(p-1)}\right)\right\rceil .
$$

If $2 \leq r \leq p-1$, then $S_{i}$ generates $V_{i}$ as an A-module, while if $r=p$, then $S_{i} \cup\left\{p^{\left\lceil\log _{p}(i)\right\rceil} w\right\}$ generates $V_{i}$ as an A-module.

Proof. Let $t=(i+1)_{m}-1$. In the case that $2 \leq r \leq p-1$, we have $\psi_{m}(i)=\psi(t)$ and $\psi(t)>0$ if and only if $\frac{i+1}{p^{m}}>r+1$, or $m<\log _{p}\left(\frac{i+1}{r+1}\right)$. The smallest $m$ such that $\psi_{m}(i)=0$ is therefore $s$. If $r=p$, then $\psi_{m}^{\prime}(i)=\psi(t)-\epsilon_{t}$, where $\epsilon_{t} \in\{0,1\}$ is 1 if and only if $p^{l+1}+1 \leq t \leq p^{l+1}+p-1$ for some $l \geq 0$. In particular, we have $\psi(t)>\epsilon_{t}$ if and only if $t \geq 2 p$, so the smallest $m$ such that $\psi_{m}^{\prime}(i)=0$ is again $s$.

It suffices to show that the images of our elements generate $V_{i} / V_{i+p-1}$. Suppose that $\alpha=\left(\rho^{k} b T^{j}+c\right) u_{r}+d w \in V_{i}$ for some nonnegative integers $j, k, b \in \mathbb{Z}_{p}[\Phi]-$ $p \mathbb{Z}_{p}[\Phi], \quad c \in T^{j+1} A$, and $d \in \mathbb{Z}_{p}[\Phi]$ (with $d=0$ if $r \neq p$ ). Let $m=\min (k, s)$. Then $j \geq \psi_{m}^{\prime}(i)$ by Theorems 3.2.2 and 3.2.4 and Corollary 3.3.2 (and the fact that $\left.\psi_{s}^{\prime}(i)=0\right)$, and we set

$$
\alpha^{\prime}=\alpha-\rho^{k-m} b T^{j-\psi_{m}^{\prime}(i)} \kappa_{m, i} \in V_{i} \cap\left(A\left(T^{j+1} u_{r}, w\right)\right) .
$$

If $r \leq p-1$, we may repeat this process recursively until we obtain an element of $V_{i+p-1}$. If $r=p$, either $\kappa_{m, i} \in A u_{p}$ or $\kappa_{m, i} \in \rho^{m+l+1} w+A u_{p}$ for some $l \geq 0$ with $i<p^{m+l+1}+p^{m+1}$. Since $(T, p) p^{m+l+1} w \subseteq V_{p^{m+l+2}}$, there exists an element

$$
\alpha^{\prime \prime} \in V_{i} \cap\left(T^{j+1} A u_{r}+\mathbb{Z}_{p}[\Phi] w\right)
$$

with $\alpha^{\prime \prime}-\alpha^{\prime} \in V_{i+p-1}$, and again we may repeat the process until we obtain an element of $V_{i+p-1}$ plus an element of $V_{i} \cap \mathbb{Z}_{p}[\Phi] w=\mathbb{Z}_{p}[\Phi] p^{\left\lceil\log _{p}(i)\right\rceil} w$.

Lemma 3.4.2. If $m \geq 1$ is such that $\theta_{m}(i) \geq 1$, then $\theta_{m-1}(i) \geq \theta_{m}(i)+2$.

Proof. First, suppose that $\theta_{m}(i) \geq 1$, and note that $i_{m-1} \geq p\left(i_{m}-1\right)+1$. Therefore,

$$
\theta_{m-1}(i) \geq \psi\left(p\left(i_{m}-1\right)+1\right)=i_{m}-1+\left\lfloor\frac{2+\left\{r-i_{m}\right\}}{p}\right\rfloor-\delta .
$$


On the other hand,

$$
\theta_{m}(i)=\left\lfloor\frac{i_{m}+1+\left\{r-i_{m}\right\}}{p}\right\rfloor-\delta .
$$

In particular, $\theta_{m}(i)=1$ exactly when $r+1 \leq i_{m} \leq r+(\delta+1)(p-1)$. In this case,

$$
\theta_{m-1}(i) \geq r+1-\delta \geq 3=\theta_{m}(i)+2 \text {. }
$$

In general, (3.4.1) and (3.4.2) tell us that

$$
\theta_{m-1}(i) \geq i_{m}-1-\delta \quad \text { and } \quad \frac{i_{m}}{p}+1-\delta \geq \theta_{m}(i)
$$

and we have

$$
i_{m}-1-\delta \geq \frac{i_{m}}{p}+3-\delta
$$

if and only if $i_{m} \geq \frac{4 p}{p-1}$, which holds for $i_{m} \geq r+p$ unless $i_{m}=5, r=2$, and $p=3$, in which case $\theta_{m-1}(i) \geq 5$ and $\theta_{m}(i)=2$.

For each $m \geq 0$, let us set $\epsilon_{m}(i)=\theta_{m}(i)-\psi_{m}^{\prime}(i)$, which lies in $\{0,1\}$ by Lemma 3.3.4 and the remark before it. The following corollary is useful in understanding the form of our special elements.

Corollary 3.4.3. For every $m \geq 0$, we have $\psi_{m}^{\prime}(i) \geq \psi_{m+1}^{\prime}(i)$, with equality if and only if $\psi_{m}^{\prime}(i)=0$.

Proof. If $\theta_{m+1}(i) \geq 1$, Lemma 3.4.2 and the fact that $\epsilon_{k}(i) \in\{0,1\}$ for all $k$ imply that $\psi_{m}^{\prime}(i)>\psi_{m+1}^{\prime}(i)$. Otherwise, $\psi_{m+1}^{\prime}(i)=0$, and the inequality holds automatically, with equality exactly if $\psi_{m}^{\prime}(i)=0$.

We next show that the sets given in Theorem 3.4.1 are minimal unless $r=p$. It is in the proof of this result that the refined elements $\kappa_{m, i}$ first hold an advantage of ease of use over the elements of Section 3.2.

Theorem 3.4.4. For $r \leq p-1$, no proper subset of $S_{i}$ generates $V_{i}$ as an A-module. For $r=p$, every proper subset of $S_{i} \cup\left\{p^{\left\lceil\log _{p}(i)\right\rceil} w\right\}$ that generates $V_{i}$ as an A-module must contain $S_{i}$.

Proof. Assume first that $2 \leq r \leq p-1$. Suppose that

$$
\sum_{m=0}^{s} c_{m} \kappa_{m, i}=0,
$$

where $c_{m} \in A$ for $m \leq s$. We must show that no $c_{m}$ is a unit. We prove the somewhat stronger claim that $c_{m} \in\left(p, T^{\epsilon_{m}(i)+1}\right)$ for each $m$.

Fix a nonnegative integer $m \leq s$. If $\epsilon_{m}(i)=0$, then $\kappa_{m, i}=\rho^{m} T^{\theta_{m}(i)} u_{r}$ by (3.3.5). If $\epsilon_{m}(i)=1$, then (3.3.6) tells us that

$$
\kappa_{m, i} \equiv \rho^{m} T^{\theta_{m}(i)-1} u_{r} \bmod A T^{\theta_{m}(i)+1} u_{r},
$$


noting Lemma 3.4.2. Set

$$
X_{m}=\left\{k \in \mathbb{Z} \mid m<k \leq s, \epsilon_{k}(i)=1, \sigma(k, i) \leq m\right\},
$$

which is actually a set of cardinality at most one, though we do not need this fact. Let $k \leq s$. If $k \in X_{m}$, then (3.3.6) and Lemma 3.4.2 together imply that

$$
\kappa_{k, i} \equiv-a_{k, i} \rho^{m} T^{\theta_{m}(i)-1} u_{r} \bmod \left(p^{m+1}, T^{\theta_{m}(i)+1}\right) u_{r},
$$

and if $k \notin X_{m}$, they and (3.3.5) similarly imply that $\kappa_{k, i} \in\left(p^{m+1}, T^{\theta_{m}(i)+1}\right) u_{r}$. Thus, (3.4.3) yields the congruence

$$
c_{m} \rho^{m} T^{\psi_{m}(i)} \equiv \sum_{k \in X_{m}} c_{k} a_{k, i} \rho^{m} T^{\theta_{m}(i)-1} \bmod \left(p^{m+1}, T^{\theta_{m}(i)+1}\right) .
$$

If the claim holds for all $k>m$, then we have $c_{k} \in\left(p, T^{2}\right)$ for each $k \in X_{m}$, so $c_{m} \in\left(p, T^{\epsilon_{m}(i)+1}\right)$, as desired.

If $r=p$, a completely analogous argument shows that at most $p^{\left\lceil\log _{p}(i)\right\rceil} w$ is unnecessary for generation, if one works modulo $A w=\mathbb{Z}_{p}[\Phi] w+A(\varphi-1) u_{p}$ throughout. Here, one should replace $X_{m}$ by

$$
X_{m}^{\prime}=\left\{k \in \mathbb{Z} \mid m<k \leq s, \epsilon_{k}(i)=1, \sigma^{\prime}(k, i) \leq m\right\},
$$

where we set $\sigma^{\prime}(k, i)=\sigma(k, i)$ unless $i_{k+1}=p^{l}+1$ for some $l \geq 0$, in which case we set $\sigma^{\prime}(k, i)=\sigma(k+1, i)$.

For the purpose of completeness, we also give the precise condition on $i$ under which no proper subset of $S_{i} \cup\left\{p^{\left\lceil\log _{p}(i)\right\rceil} w\right\}$ generates $V_{i}$ in the case that $r=p$.

Proposition 3.4.5. For $r=p$, the set $S_{i}$ generates $V_{i}$ if and only if $i_{s}=p+1$.

Proof. To determine whether $p^{\left\lceil\log _{p}(i)\right\rceil} w$ is or is not necessary, we work in distinct ranges of $i$ separately. Note that the definition of $s$ forces $2 p^{s}<i<2 p^{s+1}$.

Case 1: $2 p^{s}<i \leq p^{s+1}$. In this case, all of the elements $\kappa_{m, i}$ lie in $A u_{p}$, and therefore $p^{s+1} w$ is necessary.

Case 2: $p^{s+1}<i \leq p^{s+1}+p^{s}-p^{s-1}$. In this range, we have

$$
\kappa_{s, i}=\rho^{s} u_{p}+\rho^{s+1} w \quad \text { and } \quad \kappa_{s-1, i}=\rho^{s-1} T^{p-1} u_{p}+\rho^{s+1} w .
$$

Note that $(T-p) \kappa_{s, i}=\rho^{s}(T-p) u_{p}+\rho^{s+1}(\varphi-1) u_{p}=\rho^{s}(T-\rho) u_{p}$, so

$$
\rho^{s} T u_{p} \equiv \rho^{s+1} u_{p} \bmod A \kappa_{s, i} \quad \text { and } \quad \rho^{s} u_{p} \equiv-\rho^{s+1} w \bmod A \kappa_{s, i} .
$$

Applying these to $\rho \kappa_{s-1, i}$, we obtain

$$
\rho \kappa_{s-1, i} \equiv \rho^{s+p-1} u_{p}+\rho^{s+2} w \equiv\left(-\rho^{s+p}+\rho^{s+2}\right) w \bmod A \kappa_{s, i},
$$

which in particular tells us that $p^{s+2} w \in A\left(\kappa_{s-1, i}, \kappa_{s, i}\right)$. 
Case 3. $p^{s+1}+p^{s}-p^{s-1}<i \leq p^{s+1}+p^{s}$. In this range, we have

$\kappa_{s, i}=\rho^{s} u_{p}+\rho^{s+1} w \quad$ and $\quad \kappa_{s-1, i}=\left(\rho^{s-1} T^{p-1}+\sum_{k=\sigma(s, i)}^{s-2} \rho^{k} T^{\theta_{k}(i)-1}\right) u_{p}+\rho^{s+1} w$ with $\sigma(s, i) \leq s-2$. Moreover, $\kappa_{m, i} \in A u_{r}$ for $m \leq s-2$.

Set $v_{k, i}=\rho^{k} T^{\theta_{k}(i)} u_{r}$ for all nonnegative $k$. We note that $v_{m, i} \in A\left(\kappa_{0, i}, \ldots, \kappa_{m, i}\right)$ for $m \leq s-2$ : If $\kappa_{m, i} \neq v_{m, i}$, which is to say $\epsilon_{m}(i)=1$, then

$$
v_{m, i}=T \kappa_{m, i}+a_{m, i} \sum_{k=\sigma(m, i)}^{m-1} v_{k, i} .
$$

Let $j=\theta_{s-2}(i)-p$, and note that $j \geq p^{2}-1 \geq 2$. Since

$$
T^{j} \kappa_{s-1, i} \equiv \rho^{s-1} T^{\theta_{s-2}(i)-1} u_{p}+\rho^{s+1} T^{j} w \bmod A\left(v_{\sigma(s, i), i}, \ldots, v_{s-2, i}\right)
$$

and $\rho^{k+1} T^{\theta_{k}(i)-1} u_{p} \in A v_{k+1, i}$ for all $k$ with $\sigma(s, i) \leq k \leq s-3$, we therefore have

$$
\left(\rho-T^{j}\right) \kappa_{s-1, i} \equiv \rho^{s} T^{p-1} u_{p}+\rho^{s+1}\left(\rho-T^{j}\right) w \bmod A\left(v_{\sigma(s, i), i}, \ldots, v_{s-2, i}\right) .
$$

Using (3.4.7) to reduce (3.4.8), we see that

$$
\left(\rho-T^{j}\right) \kappa_{s-1, i} \equiv \rho^{s+2}\left(1-\rho^{p-2}-\rho^{j-1}\right) w \bmod A\left(v_{\sigma(s, i), i}, \ldots, v_{s-2, i}, \kappa_{s, i}\right),
$$

which implies that $p^{s+2} w \in A\left(\kappa_{0, i}, \ldots, \kappa_{s, i}\right)$.

Case 4: $p^{s+1}+p^{s}<i<2 p^{s+1}$. In this case, all of the $\kappa_{m, i}$ with $m \leq s-1$ lie in $A T^{p} u_{p}$, and so for $p^{s+2} w$ to be unnecessary, there would have to exist $c \in A$ such that

$$
c \kappa_{s, i} \equiv p^{s+2} w \bmod A T^{2} u_{p} .
$$

Note that $c \kappa_{s, i} \equiv c\left(\rho^{s} u_{p}+\rho^{s+1} w\right) \bmod A T^{2} u_{p}$, which forces $c \equiv T^{2} c^{\prime} \bmod (\varphi-1)$ for some $c^{\prime} \in A$. This means that

$$
c \kappa_{s, i} \equiv c^{\prime} p^{s+3} w \bmod A\left(u_{p},(\varphi-1) w\right),
$$

but $p^{s+2} w \notin A\left(p^{s+3} w,(\varphi-1) w, u_{p}\right)$, so (3.4.9) cannot hold.

\section{The finite level}

4.1. Norms and eigenspace structure. In this section, we explore the consequences of the results of Section 3 for unit groups of actual abelian local fields of characteristic 0 . Fix a positive integer $n$. Recall from the introduction that $F_{n}$ is the field obtained from $E$ by adjoining the $p^{n}$ th roots of unity and that $U_{n, t}$ denotes the $t$ th unit group of $F_{n}$ for $t \geq 1$. As before, we set $\Gamma_{n}=\operatorname{Gal}\left(F_{n} / F_{1}\right)$. 
For positive integers $m \geq n$, let $N_{m, n}$ and $\operatorname{Tr}_{m, n}$ denote, respectively, the norm and trace from $F_{m}$ to $F_{n}$. We also let $N_{n}$ denote the restriction map $N_{n}: F^{\times} \rightarrow F_{n}^{\times}$on norm compatible sequences. Recall that $\lambda_{n}=N_{n}(\lambda)=1-\zeta_{p^{n}}$, where $\zeta_{p^{n}}=N_{n}(\zeta)$ is a primitive $p^{n}$ th root of unity. We require a few preliminary lemmas.

Lemma 4.1.1. One has

$$
\operatorname{Tr}_{n+1, n}\left(\lambda_{n+1}^{p k-\epsilon}\right) \equiv p \lambda_{n}^{k-\epsilon} \bmod p^{3}
$$

for all $k \geq 1$ and $\epsilon \in\{0,1\}$.

Proof. An easy calculation shows that

$$
\operatorname{Tr}_{n+1, n}\left(\lambda_{n+1}^{t}\right)=p \sum_{j=0}^{\left\lfloor\frac{t}{p}\right\lrcorner}\left(\begin{array}{c}
t \\
p j
\end{array}\right)\left(-\zeta_{p^{n}}\right)^{j}
$$

for every $t \geq 0$. The result follows since

$$
\left(\begin{array}{c}
p k-\epsilon \\
p j
\end{array}\right)=\left(\begin{array}{c}
k-\epsilon \\
j
\end{array}\right) \prod_{\substack{s=1 \\
p \nmid s}}^{p(k-j)}\left(1+\frac{p j}{s}\right) \equiv\left(\begin{array}{c}
k-\epsilon \\
j
\end{array}\right) \bmod p^{2} \quad \text { for any } j \geq 0 .
$$

Let $e_{n}=p^{n-1}(p-1)$ denote the ramification index of $E$. In applying Lemma 4.1.1, it is useful to make note of the fact that

$$
p \equiv-\lambda_{n}^{e_{n}} \bmod \lambda_{n}^{p^{n}} .
$$

Lemma 4.1.2. For $t \geq 1$ and any unit $\eta$ in $E$, one has

$$
\begin{aligned}
& N_{n+1, n}\left(1+\eta \lambda_{n+1}^{t}\right) \\
& \equiv \begin{cases}1+\eta^{p} \lambda_{n}^{t} \bmod \lambda_{n}^{t+1} & \text { if } t<p^{n}-1, \\
1+\left(\eta^{p}-\eta\right) \lambda_{n}^{p^{n}-\epsilon} \bmod \lambda_{n}^{p^{n}+1-\epsilon} & \text { if } t=p^{n}-\epsilon, \epsilon \in\{0,1\}, \\
1-\eta \lambda_{n}^{e_{n}+k-\epsilon} \bmod \lambda_{n}^{e_{n}+k+1-\epsilon} & \text { if } t=p k-\epsilon>p^{n}, \epsilon \in\{0,1\} .\end{cases}
\end{aligned}
$$

Moreover, we have

$$
N_{n+1, n}\left(1+\eta \lambda_{n+1}^{t}\right) \equiv 1 \bmod \lambda_{n}^{e_{n}+\lfloor t / p\rfloor}
$$

for all $t>p^{n}$.

Proof. The jump in the ramification filtration of $\operatorname{Gal}\left(F_{n+1} / F_{n}\right)$ occurs at $p^{n}-1$. By [Serre 1979, Lemmas V.4 and V.5], we have

$$
\begin{aligned}
N_{n+1, n}\left(1+\eta \lambda_{n+1}^{t}\right) & \equiv 1+\eta \operatorname{Tr}_{n+1, n}\left(\lambda_{n+1}^{t}\right)+\eta^{p} \lambda_{n}^{t} \bmod \lambda_{n}^{e_{n}+\lfloor 2 t / p\rfloor}, \\
\operatorname{Tr}_{n+1, n}\left(\lambda_{n+1}^{t}\right) & \equiv 0 \bmod \lambda_{n}^{e_{n}+\lfloor t / p\rfloor} .
\end{aligned}
$$

The result is then a corollary of Lemma 4.1.1, upon applying (4.1.1). 
Let $D_{n}$ be the pro- $p$ completion of $F_{n}^{\times}$, and let $D_{n}^{(r)}=D_{n}^{\varepsilon_{r}}$ for any $r \in \mathbb{Z}$. As before, we fix $r$ with $2 \leq r \leq p$, and $i$ will always denote a positive integer with $i \equiv r \bmod p-1$. Let $V_{n, i}=U_{n, i}^{\varepsilon_{r}}=U_{n, i} \cap D_{n}^{(r)}$ for any such $i$. These $V_{n, i}$ are all modules over $A_{n}=\mathbb{Z}_{p}\left[\Gamma_{n} \times \Phi\right]$. As in Lemma 2.1, we have isomorphisms

$$
V_{n, i} / V_{n, i+p-1} \stackrel{\sim}{\longrightarrow} \mathbb{F}_{q}
$$

that send $1+x \lambda_{n}^{i}$ for some $x$ in the valuation ring of $F_{n}$ to the element $\bar{x}$ of $\mathbb{F}_{q}$ that is identified with the image of $x$ in the residue field of $F_{n}$ under the isomorphism fixed in Section 2. We may then set $V_{n, i}^{\prime}=V_{n, i}-V_{n, i+p-1}$ and define $V_{n, i}(\eta)$ for $\eta \in \mathbb{F}_{q}^{\times}$as the set of elements $1+x \lambda_{n}^{i}$ with $\bar{x}=\eta$.

We have the following consequence of Lemma 4.1.2.

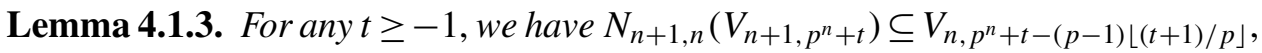
with equality for $t \geq 0$.

Proof. Note that Lemma 4.1.2 yields $N_{n+1, n}\left(U_{n+1, p^{n}+p k-\epsilon}\right)=U_{n, p^{n}+k-\epsilon}$ for all $k \geq 0$ and $\epsilon \in\{0,1\}$ with $k \geq \epsilon$, since every element in $U_{n, p^{n}+k-\epsilon}$ can be written as a product of elements of the form $1+\eta_{t} \lambda_{n}^{p^{n}+t}$ with $t \geq k-\epsilon$ and $\eta_{t} \in \mathbb{F}_{q}$. (For $k=0$ and $\epsilon=-1$, it tells us just that any element of $U_{n+1, p^{n}-1}$ has a norm in $U_{n, p^{n}-1}$.)

Note that

$U_{n, p^{n}+k-\epsilon}^{\varepsilon_{r}}=V_{n, p^{n}+k-\epsilon+\{r-k+\epsilon-1\}} \quad$ and $\quad U_{n+1, p^{n}+p k-\epsilon}^{\varepsilon_{r}}=V_{n, p^{n}+p k-\epsilon+\{r-k+\epsilon-1\}}$.

For any $t \geq 0$, we may write $t=p k-\epsilon+\{r-k+\epsilon-1\}$ for some $k, \epsilon$, and $r$, and we have

$$
t-(p-1)\left\lfloor\frac{t+1}{p}\right\rfloor=k-\epsilon+\{r-k+\epsilon-1\}
$$

The next corollary is almost immediate from Lemmas 4.1.2 and 4.1.3, so we leave it to the reader.

Corollary 4.1.4. For any unit $\eta$ in $E$, one has

$$
N_{n+1, n}\left(V_{n+1, i}(\eta)\right) \subseteq \begin{cases}V_{n, i}\left(\eta^{p}\right) & \text { if } i<p^{n}-1, \\ V_{n, i}\left(\eta^{p}-\eta\right) & \text { if } i=p^{n}-1, \\ V_{n, p^{n}+k-1}(-\eta) & \text { if } i=p^{n}+p k-1 \text { for some } k>0,\end{cases}
$$

with equality if $r \neq p-1$ or $i>p^{n}$.

As for the $p$-power map, we have a well-known and easy-to-prove fact:

Lemma 4.1.5. Suppose that $i>p^{n-1}$. Then the pth power map induces an isomorphism $V_{n, i} \stackrel{\sim}{\longrightarrow} V_{n, i+e_{n}}$, and we have $V_{n, i}(\eta)^{p}=V_{n, i+e_{n}}(-\eta)$ for all $\eta \in \mathbb{F}_{q}^{\times}$.

Next, we discuss the restriction map from the field of norms to the finite level. 
Proposition 4.1.6. The map $N_{n}$ induces maps $N_{n}: D^{(r)} \rightarrow D_{n}^{(r)}$ that are surjections for $r \neq p-1$ and which have procyclic cokernel for $r=p-1$. For $\eta \in \mathbb{F}_{q}^{\times}$, we have

$$
N_{n}\left(V _ { i } ( \eta ) \subseteq \subseteq \left\{\begin{array}{ll}
V_{n, i}\left(\eta^{p^{-n}}\right) & \text { if } i \leq p^{n}-2, \\
V_{n, p^{n}-1}\left(\eta^{p^{-n}}-\eta^{p^{-n-1}}\right) & \text { if } i=p^{n}-1, \\
V_{n, p^{n}+k-1}\left(-\eta^{p^{-n-1}}\right) & \text { if } i=p^{n}+p k-1 \text { for some } 0<k<e_{n} .
\end{array}\right.\right.
$$

Moreover, we have induced maps $V_{i} / V_{i+1} \rightarrow V_{n, i} / V_{n, i+1}$ for all $i<p^{n}$, and these are isomorphisms for $i \neq p^{n}-1$. For $i \leq p^{n}$, we have $V_{n, i}=N_{n} V_{i}$ if $r \neq p-1$, and $V_{n, i} / N_{n} V_{i}$ is procyclic if $r=p-1$.

Proof. That the cokernel of $N_{n}$ is trivial if $r \neq p-1$ and procyclic if $r=p-1$ follows easily from local class field theory, but it is also a consequence of the argument that follows. The first jump in the ramification filtration of $\mathrm{Gal}\left(F_{\infty} / F_{n+1}\right)$ is at $p^{n+1}-1$. In particular, for $t$ less than this value, repeated application of Lemma 4.1.2 tells us

$$
N_{n+1}\left(1+\eta \lambda^{t}\right)=\lim _{m \rightarrow \infty} N_{m, n+1}\left(1+\eta^{p^{-m}} \lambda_{m}^{t}\right) \equiv 1+\eta^{p^{-n-1}} \lambda_{n+1}^{t} \bmod \lambda_{n+1}^{t+1} .
$$

Moreover, repeated application of Corollary 4.1.4 followed by two applications of Lemma 4.1.3 tells us that $N_{n}\left(V_{p^{n+1}-1+\{r\}}\right) \subseteq V_{n, p^{n}+e_{n}-1+\{r\}}$. An application of Corollary 4.1.4 then yields the stated containments.

Since $\eta^{p^{-n}}$ and $-\eta^{p^{-n-1}}$ run through all elements of $\mathbb{F}_{q}$ as $\eta \in \mathbb{F}_{q}$ varies, we obtain $V_{n, i}=N_{n} V_{i}+V_{n, i+p-1}$ for all $i \leq p^{n}$ but $p^{n}-1$. Noting Lemma 4.1.5, this implies

$$
V_{n, i+k e_{n}}=N_{n} V_{p^{k} i}+V_{n, i+k e_{n}+p-1}
$$

for $p^{n-1}<i \leq p^{n}$ with $i \neq p^{n}-1$ and $k \geq 0$. Note that every element of every $V_{n, i}$ may be written as an infinite product over $j \geq 0$ of one element from each of a fixed set of representatives of the $V_{n, i+j(p-1)} / V_{n, i+(j+1)(p-1)}$. Thus, we have $N_{n} V_{i}=V_{n, i}$ so long as $r \neq p-1$.

If $r=p-1$, we can choose an element $z_{n}$ of $V_{n, p^{n}-1}(\xi)$ that is not a norm. By the formula proven above for $N_{n}\left(1+\eta \lambda^{p^{n}-1}\right)$ modulo $\lambda_{n}^{p^{n}}$, we have

$$
V_{n, p^{n}+k e_{n}-1}=N_{n} V_{p^{k}\left(p^{n}-1\right)}+V_{n, p^{n}+k e_{n}+p-2}+\left\langle z_{n}^{p^{k}}\right\rangle
$$

for $k=0$, and then for all $k \geq 0$ by taking powers. Therefore, $V_{n, i} / N_{n} V_{i}$ is generated by $z_{n}$ for all $i<p^{n}$ with $i \equiv 0 \bmod p-1$.

The following structural result is again essentially found in [Greither 1996], without the stated congruences. Here, we derive it from more basic principles.

Theorem 4.1.7. For $r \leq p-2$, the $A_{n}$-module $D_{n}^{(r)}$ is freely generated as an $A_{n}$-module by an element $u_{n, r} \in V_{n, r}(\xi)$. The $A_{n}$-module $D_{n}^{(p-1)}$ has a presentation

$$
D_{n}^{(p-1)}=\left\langle\pi_{n}, u_{n, p-1}, v_{n} \mid \pi_{n}^{\varphi}=\pi_{n}, \pi_{n}^{\gamma-1}=u_{n, p-1}^{N_{\Phi}}, v_{n}^{\gamma}=v_{n}, u_{n, p-1}^{N_{\Gamma_{n}}}=v_{n}^{1-\varphi}\right\rangle,
$$


where $v=v_{n}^{\varphi^{2-n}} \equiv 1+p \xi \bmod p^{2}$ is independent of $n$ and $u_{n, p-1} \in V_{n, p-1}(\xi)$ for $n \geq 2$, while $u_{1, p-1} \in V_{n, p-1}\left(\xi-\xi^{p^{-1}}\right)$. The $A_{n}$-module $D_{n}^{(p)}$ has a presentation

$$
D_{n}^{(p)}=\left\langle u_{n, p}, w_{n} \mid w_{n}^{\gamma-1-p}=u_{n, p}^{\varphi-1}\right\rangle
$$

with $u_{n, p} \in V_{n, p}(\xi)$ and $w_{n} \in V_{n, 1}(-\xi)$ such that $w_{n}^{N_{\Phi}}=\zeta_{p^{n}}$.

Proof. We set $u_{n, r}=\left(N_{n} u_{r}\right)^{\varphi^{n}}, \pi_{n}=N_{n} \pi$, and $w_{n}=\left(N_{n} w\right)^{\varphi^{n}}$ with $u_{r}$, $\pi$, and $w$ as in Theorem 3.1.5. It follows from the surjectivity of $N_{n}$ for $r \neq p-1$ in Proposition 4.1.6 that the element $u_{n, r}$ generates $D_{n}^{(r)}$ for $r \leq p-2$, while the elements $w_{n}$ and $u_{n, r}$ generate $D_{n}^{(p)}$. By Hilbert's Theorem 90 , the kernel of $N_{n}$ consists exactly of elements of the form $\alpha^{\gamma^{p^{n-1}}-1}$ with $\alpha \in D$, and therefore it follows that $D_{n}^{(r)}$ is free of rank 1 on $u_{n, r}$ over $A_{n}$ for $r \leq p-2$ and that $D_{n}^{(p)}$ has the stated presentation. (That $u_{1, p} \in V_{1, p}(\xi)$ requires a simple check using Propositions 3.1.3 and 4.1.6.)

The elements $\pi_{n}$ and $u_{n, p-1}$ automatically satisfy the first two relations in the desired presentation of $D_{n}^{(p-1)}$. In particular,

$$
u_{n, p-1}^{N_{\Gamma_{n} \times \Phi}}=\pi_{n}^{(\gamma-1) N_{\Gamma_{n}}}=1
$$

so Hilbert's Theorem 90 tells us that $u_{n, p-1}^{N_{\Gamma_{n}}}=v_{n}^{1-\varphi}$ for some $v_{n}$ in the pro- $p$ completion of $E^{\times}$. By Proposition 4.1.6, we have

$$
u_{n, p-1}^{N_{\Gamma_{n}}} \equiv 1+\left(\xi^{p^{n-1}}-\xi^{p^{n-2}}\right) \lambda_{1}^{p-1} \bmod \lambda_{1}^{p} \text {. }
$$

Noting (4.1.1), we may in fact choose $v_{n} \equiv 1+p \varphi^{n-2}(\xi) \bmod p^{2}$ with $v=v_{n}^{\varphi^{2-n}}$ independent of $n$.

Hilbert's Theorem 90 and Theorem 3.1.5 tell us that the $A_{n}$-module generated by $u_{n, p-1}$ is isomorphic to $A_{n} /\left(N_{\Gamma_{n} \times \Phi}\right)$. By Proposition 4.1.6, the cokernel of $N_{n}$ on $D^{(p-1)}$ is isomorphic to $\mathbb{Z}_{p}$. We claim that the image of $v$ topologically generates this cokernel. If this is the case, then clearly $D_{n}^{(p-1)}$ is generated by $\pi_{n}, u_{n, p-1}$, and $v$, and any solution with $b, d \in \mathbb{Z}_{p}$ and $c \in A_{n}$ to $\pi_{n}^{b} u_{n, p-1}^{c} v^{d}=1$ must satisfy $b=d=0$ and $c \in \mathbb{Z}_{p} N_{\Gamma_{n} \times \Phi}$.

It remains only to demonstrate the claim. Suppose by way of contradiction that there exists $a \in A_{n}$ such that $x=v u_{n, p-1}^{a}$ is a $p$ th power in $D_{n}^{(p-1)}$. This implies that $x^{\gamma-1}=u_{n, p-1}^{a(\gamma-1)}$ is a $p$ th power in the $A_{n}$-module generated by $u_{n, p-1}$. It follows that $a(\gamma-1) \in A_{n}\left(p, N_{\Gamma_{n} \times \Phi}\right)$, which forces $a(\gamma-1) \equiv 0 \bmod p$, so $a \in A_{n}\left(p, N_{\Gamma_{n}}\right)$. It then suffices to show that

$$
v u_{n, p-1}^{b N_{\Gamma_{n}}}=v^{1+b \varphi^{n-2}(1-\varphi)}
$$

is not a $p$ th power in $F_{n}$ for any $b \in \mathbb{Z}_{p}[\Phi]$. If it were for some $b$, then $v^{N_{\Phi}}$ and hence $1+p$ would be a $p$ th power in $F_{n}$ as well, but this is clearly not the case. 
4.2. Special elements. We assume for the rest of the paper that $n \geq 2$, the case that $n=1$ being slightly exceptional but also completely straightforward. In this subsection, we construct special elements in the groups in the unit filtration of $F_{n}^{\times}$. Aside from the case that $r=p-1$, these arise as restrictions of the elements introduced in Section 3.2.

Note that $\mathbb{Z}_{p}\left[\Gamma_{n}\right] \cong \mathbb{Z}_{p}[T] /\left(f_{n}\right)$, where $f_{n}=(T+1)^{p^{n-1}}-1$. Of course, we can then speak of the action of $T$ on an element of $D_{n}^{(r)}$. Once again reverting to additive notation, the following is now an immediate corollary of Theorem 3.2.2 and Proposition 4.1.6.

Proposition 4.2.1. Let $m$ and $j$ be nonnegative integers with $\phi_{m}(j)<p^{n}-1$. Define

$$
\alpha_{n, m, j}=\frac{1}{[r] !}\left(\{r-\delta-j\} ! \rho^{m} T^{j}-\sum_{k=1}^{m} \rho^{m-k} T^{\phi_{k-1}(j)-\delta}\right) u_{n, r},
$$

unless $j=0$ and $r=p-1$, in which case we replace $\{r-\delta-j\}$ ! with -1 in the formula. Then $\alpha_{n, m, j} \in V_{n, \phi_{m}(j)}(\xi)$. Furthermore, $\left(p^{m} b T^{j}+c\right) u_{n, r} \notin V_{n, \phi_{m}(j)+p-1}$ for all $b \in \mathbb{Z}_{p}[\Phi]-p \mathbb{Z}_{p}[\Phi]$ and $c \in T^{j+1} A_{n}$.

For nonnegative $m \leq n-2$, define $\phi_{n, m}^{\prime}: \mathbb{Z}_{\geq 0} \rightarrow \mathbb{Z}_{\geq 0}$ by $\phi_{n, m}^{\prime}(j)=\phi_{m}^{\prime}(j)$ unless $r=p-1$ and $j=e_{n-m-1}$, in which case we set

$$
\phi_{n, m}^{\prime}\left(e_{n-m-1}\right)=e_{n}+p^{m+1}-1=\phi_{m}\left(e_{n-m-1}\right)+p^{m}(p-1) .
$$

For nonnegative $k$, define $\vartheta_{2, k}=1+\varphi^{-1}+\cdots+\varphi^{-k}$ and $\vartheta_{j, k}=1$ for $j>2$. Note that $\vartheta_{2, k} \in p \mathbb{Z}_{p}[\Phi]$ if and only if $k \equiv-1 \bmod p|\Phi|$.

By Theorem 4.1.7, every element of $V_{n, p-1}$ may be written as $c u_{n, p-1}+d v$ with $c \in A_{n}$ and $d \in \mathbb{Z}_{p}$, and this representation is unique up to the choice of $c$ modulo $N_{\Gamma_{n} \times \Phi}$. For $a, b \in D_{n}^{(r)}$, we again write $a \sim b$ if $a, b \in V_{n, i}(\eta)$ for some $i$ and $\eta \in \mathbb{F}_{q}^{\times}$.

Theorem 4.2.2. Let $m \leq n-2$ be a nonnegative integer, and define

$$
\omega_{n, m}=\sum_{k=0}^{m} \rho^{m-k} \vartheta_{n-m, k} T^{p^{n-m+k-2}(p-1)+p^{k}-1} u_{n, p-1}-v .
$$

Then we have $\omega_{n, m} \in V_{n, e_{n}+p^{m+1}-1}(\xi)$. Furthermore, if $j \geq 0$ with $\phi_{m}(j)<p^{n}$, then

$$
\left(p^{m} T^{j} b+c\right) u_{n, p-1}+d v \notin V_{\phi_{n, m}^{\prime}(j)+p-1}
$$

for all $b \in \mathbb{Z}_{p}[\Phi]-p \mathbb{Z}_{p}[\Phi], c \in T^{j+1} A_{n}$, and $d \in \mathbb{Z}_{p}$.

Proof. Let $l$ be a nonnegative integer with $l \leq m$. We define

$$
\omega_{n, m, l}=\sum_{k=0}^{l} \rho^{m-k} \vartheta_{n-m, k} T^{p^{n-m+k-2}(p-1)+p^{k}-1} u_{n, p-1}-v .
$$


We claim not only that $\omega_{n, m}=\omega_{n, m, m} \in V_{n, e_{n}+p^{m+1}-1}(\xi)$, but that, for $l<m$,

$$
\omega_{n, m, l} \in \begin{cases}V_{n, e_{n}+p^{m+1}-p^{m-l}}\left(\vartheta_{n-m, l+1} \xi\right) & \text { if } p \nmid \vartheta_{n-m, l+1}, \\ V_{n, e_{n}+p^{m+1}-p^{m-l-1}}(\xi) & \text { if } p \mid \vartheta_{n-m, l+1} .\end{cases}
$$

We note, to begin with, that $\omega_{n, m, l} \in V_{n, e_{n}+p-1}$, since Lemma 3.2.1a implies

$$
\omega_{n, m, l}+v \sim \rho^{m} T^{e_{n-m-1}} u_{n, p-1} \in V_{n, e_{n}}(-\xi)
$$

For a given $i$, we take $V_{n, i}(0)$ to mean $V_{n, i+p-1}$ in what follows.

If $p \nmid \vartheta_{n-m, l}$, then Lemmas 3.2.1a and 4.1.5 imply that

$$
T \omega_{n, m, l} \sim \rho^{m-l} \vartheta_{n-m, l} T^{p^{n-m+l-2}(p-1)+p^{l}} u_{n, p-1}
$$

if $l<m, m=0$, or $m<n-2$, and we have

$$
T \omega_{n, m, l} \in \begin{cases}V_{n, e_{n}+p^{m+1}+p^{m-l}(p-2)}(-\xi) & \text { if } m<n-2 \text { or } m=0, \\ V_{n, p^{n}+p^{m-l-1}(p-2)}\left(\vartheta_{2, l} \varphi^{-1} \xi\right) & \text { if } l<m=n-2 .\end{cases}
$$

On the other hand, if $p \mid \vartheta_{n-m, l}$, then we have $T \omega_{n, m, l} \sim T \omega_{n, m, l-1}$, so we can still apply (4.2.2). Moreover, since $\vartheta_{2, n-3} \varphi^{-1}-\vartheta_{2, n-2}=-1$ for $n \geq 3$, we have

$$
T \omega_{n, n-2} \sim \rho \vartheta_{2, n-3} T^{p^{n-3}} u_{n, p-1}+\vartheta_{2, n-2} T^{p^{n-2}} u_{n, p-1} \in V_{n, p^{n}+p-2}(-\xi) .
$$

We prove our claim by induction on $m$. In the case that $m=0$, we have that $T \omega_{n, 0} \in V_{n, e_{n}+2 p-2}(-\xi)$ by (4.2.2), and we have seen that $\omega_{n, 0} \in V_{n, e_{n}+p-1}$, so Proposition 2.5 forces $\omega_{n, 0} \in V_{n, e_{n}+p-1}(\xi)$. For $m \geq 1$, that $\omega_{n, m} \in V_{n, e_{n}+p^{m+1}-1}$ follows from the claim for $l=m-1$ and the fact that

$$
\omega_{n, m}-\omega_{n, m, m-1}=\vartheta_{n-m, m} T^{e_{n-1}+p^{m}-1} u_{n, p-1}
$$

is an element of $V_{n, e_{n}+p^{m+1}-p}\left(-\vartheta_{n-m, m} \xi\right)$. Since $T \omega_{n, m} \in V_{n, e_{n}+p^{m+1}+p-2}(-\xi)$, an application of Proposition 2.5 would then yield that $\omega_{n, m} \in V_{n, e_{n}+p^{m+1}-1}(\xi)$. So, to perform the inductive step for $l<m$, we assume that either $p \nmid \vartheta_{n-m, l+1}$ or $l=m-1$, since otherwise $\omega_{n, m, l} \sim \omega_{n, m, l+1}$ and $l+1<m$.

By Lemma 4.1.5 and induction, we have $N_{n, n-1}\left(\omega_{n, m, l}\right)$

$$
=p \omega_{n-1, m-1, l} \in \begin{cases}V_{n-1,2 e_{n-1}+p^{m}-p^{m-l-1}}\left(-\vartheta_{n-m, l+1} \xi\right) & \text { if } l<m-1, \\ V_{n-1,2 e_{n-1}+p^{m}-p^{m-l-1}}(-\xi) & \text { if } l=m-1 .\end{cases}
$$

Let $i$ be such that $\omega_{n, m, l} \in V_{n, i}^{\prime}$, and set $t=e_{n}+p^{m+1}-p^{m-l}$. By Lemma 4.1.3, we have both that $i \leq t+p-1$ and that there exists $x \in V_{n, t}^{\prime}$ with $N_{n, n-1}(x)=p \omega_{n-1, m-1, l}$. Hilbert's Theorem 90 implies that $x-\omega_{n, m, l} \in A_{n} f_{n-1} u_{n, p-1}$. Note that

$$
p f_{n-1} u_{n, p-1} \sim p T^{p^{n-2}} u_{n, p-1} \in V_{n, p^{n}+p-2}
$$

while $\omega_{n, m, l} \notin V_{n, p^{n}+p-2}$. It follows that

$$
x \sim \omega_{n, m, l}+b T^{g} u_{n, p-1}
$$


for some $b \in A_{n}$ with $b \notin(p, T)$ and $g \geq p^{n-2}$. Since $b T^{g+1} u_{n, p-1} \in V_{n, \phi(g+1)}^{\prime}$ by Lemma 3.2.1a and both $T x$ and $T \omega_{n, m, l}$ lie in $V_{n, t+p-1}$, the latter by (4.2.2), we have $\phi(g+1)>t$ and hence $\phi(g) \geq t$. Therefore, we have $b T^{g} u_{n, p-1} \in V_{n, t}$, and (4.2.4) now forces $i \geq t$, which means that $i \in\{t, t+p-1\}$.

If $l<n-3$, then Lemma 2.2 forces $i=t$ in order for (4.2.2) to hold. If $l=n-3$ and $i=t+p-1$, then Proposition 2.5 and (4.2.2) force $\omega_{n, n-2, n-3}$ to be in $V_{n, p^{n}-1}\left(-\vartheta_{2, n-3} \varphi^{-1} \xi\right)$. By Corollary 4.1.4, this implies that

$$
N_{n, n-1}\left(\omega_{n, n-2, n-3}\right) \in V_{n-1,2 e_{n-1}+p^{n-2}-1}\left(\vartheta_{2, n-3} \varphi^{-1} \xi\right),
$$

and then (4.2.3) tells us that $p \mid \vartheta_{2, n-2}$ and $\omega_{n, n-2, n-3} \in V_{n, p^{n}-1}(\xi)$.

If $i=t$, then Lemma 3.2.1a implies that

$$
\omega_{n, m, l} \sim-d T^{e_{n-1}+p^{m}-p^{m-l-1}} u_{n, p-1}
$$

for some $d \in \mathbb{Z}_{p}[\Phi]-p \mathbb{Z}_{p}[\Phi]$. Set

$$
z=\omega_{n, m, l}+d T^{e_{n-1}+p^{m}-p^{m-l-1}} u_{n, p-1} \in V_{n, t+p-1} .
$$

By (4.2.2) and Lemma 3.2.1a, we have $T z \in V_{n, t+2(p-1)}\left(-d^{\prime} \xi\right)$, where $d^{\prime}=d$ if $l<n-3$ and $d^{\prime}=d-\vartheta_{2, n-3} \varphi^{-1}$ if $l=n-3$. We therefore have $z \in V_{n, t+p-1}\left(d^{\prime} \xi\right)$, and then

$$
N_{n, n-1}(z) \in V_{n-1,2 e_{n-1}+p^{m}-p^{m-l-1}}\left(-d^{\prime} \xi\right)
$$

by Corollary 4.1.4. On the other hand, we have

$$
N_{n, n-1}\left(T^{e_{n-1}+p^{m}-p^{m-l-1}} u_{n, p-1}\right)=\varphi T^{e_{n-1}+p^{m}-p^{m-l-1}} u_{n-1, p-1} \in V_{n-1, t},
$$

so we have $N_{n, n-1}(z) \sim N_{n, n-1}\left(\omega_{n, m, l}\right)$. By (4.2.3), we then have $d=\vartheta_{n-m, l+1}$. If $p \mid \vartheta_{n-m, l+1}$, then $l=n-3$ by assumption, and this contradicts our assumption on $i$ and implies the claim for $\omega_{n, n-2, n-3}$. Otherwise, we have already shown that $i=t$, and (4.2.5) and Lemma 3.2.1a yield the claim.

Suppose now that $j \geq 0, b \in \mathbb{Z}_{p}[\Phi]-p \mathbb{Z}_{p}[\Phi], c \in T^{j+1} A_{n}$, and $d \in \mathbb{Z}_{p}$ are such that $\phi_{m}(j)<p^{n}$ and

$$
\omega=\left(p^{m} b T^{j}+c\right) u_{n, p-1}+d v \in V_{n, i}^{\prime}
$$

for some $i \geq \phi_{n, m}^{\prime}(j)$. We suppose that $\phi_{m}(j) \geq e_{n}$, as the result otherwise reduces to Proposition 4.2.1. For $m=0$, if $\left(b T^{j}+c\right) u_{n, p-1} \neq-d v$, then $i=e_{n}$ or $i=\phi(j) \leq \phi_{n, 0}^{\prime}(j)$. Otherwise, we must have $j=e_{n-1}$, and since $T \omega \sim b T^{j+1} u_{n, p-1}$, the argument of Lemma 3.2.1b tells us that $i=e_{n}+p-1$.

For $m \geq 1$, we rewrite $c$ as $p c^{\prime}+T^{h} v$ for some $h \geq j+1$ and $c^{\prime}, v \in A_{n}$ with $v \notin(p, T)$. Note that $\phi_{n, m}^{\prime}(j)=p \phi_{n-1, m-1}^{\prime}(j)+p-1$. By induction, we have

$$
\left(p^{m-1} b T^{j}+c^{\prime}\right) \varphi u_{n-1, p-1}+d v \notin V_{n-1, \phi_{n-1, m-1}^{\prime}}(j)+p-1 .
$$


The $p$ th power of this element is the norm from $F_{n}$ of

$$
\omega^{\prime}=\omega-T^{h} v u_{n, p-1}=\left(p^{m} b T^{j}+p c^{\prime}\right) u_{n, p-1}+d v,
$$

and $\omega^{\prime} \notin V_{n, \phi_{n, m}^{\prime}(j)+p-1}$ by Lemma 4.1.3. If $\omega^{\prime} \in V_{n, \phi_{n, m}^{\prime}(j)}$, then the fact that $\phi_{n, m}^{\prime}(j)$ is -1 modulo $p$ and therefore not a value of $\phi$ implies that $\omega^{\prime} \nsucc-T^{h} v u_{n, p-1}$, so we have $i=\phi_{n, m}^{\prime}(j)$.

So, assume that $\omega^{\prime} \notin V_{n, \phi_{n, m}^{\prime}(j)}$. Then $\omega^{\prime} \sim-T^{h} v u_{n, p-1}$, and Lemma 3.2.1a implies that $\phi(h)<\phi_{n, m}^{\prime}(j) \leq i$. If $\omega \notin V_{n, \phi(h+1)}$, then we must have

$$
i=\phi(h)+p-1=\phi_{n, m}^{\prime}(j) .
$$

So, we assume moreover that $\omega \in V_{n, \phi(h+1)}$, in which case $T \omega^{\prime} \sim-T^{h+1} v u_{n, p-1}$. Since $T \omega^{\prime}$ is a power of $p$, either $\phi(h+1)$ is divisible by $p$ and less than $p^{n}$, or $\phi(h+1)>p^{n}$. In the former case, unless $\phi(h+2)>p^{n}$, we would have $T^{2} \omega^{\prime} \in V_{n, \phi(h+1)+p(p-1)}$ and then $T^{2} \omega \in V_{n, \phi(h+2)}^{\prime}$, contradicting $\omega \in V_{n, \phi(h+1)}$. We therefore have $\phi(h+2)>p^{n}$ in both cases, so $T \omega^{\prime} \in V_{n, p^{n}-p}$. By Proposition 4.2.1 and the fact that $\phi_{m}\left(p^{n-m-1}\right)>p^{n}$, this forces $j=p^{n-m-1}-1$. If $m<n-2$, then

$$
p^{n}-p \leq \phi(h)+p-1 \leq \phi_{n, m}^{\prime}(j)=\phi_{m}(j)=p^{n}-p^{m+1}+p^{m}-1,
$$

which is a contradiction. We therefore have $m=n-2$ and $j=p-1$, so

$$
p^{n}-1=\phi_{n, n-2}^{\prime}(p-1)>\phi_{n-2}(p-1),
$$

which, noting Proposition 4.2.1, implies that $p \nmid d$ and then, noting Theorem 4.1.7, that $\omega \notin p D_{n}^{(p-1)}$. In particular, $\omega \notin V_{n, p^{n}+p-2}$, so $i=p^{n}-1$.

Remark 4.2.3. Note that $\phi_{n-1}(0)=p^{n}-1<p^{n}$ as well, but in this case, the element $u_{n, p-1}^{N_{\Gamma_{n} \times \Phi}}=1$ has the form $\left(p^{n-1} b+c\right) u_{n, p-1}$ with $b \in \mathbb{Z}_{p}[\Phi]-p \mathbb{Z}_{p}[\Phi]$ and $c \in T A$.

For $r=p$, the following is a consequence of Theorem 3.2.4 and Proposition 4.1.6.

Proposition 4.2.4. Let $m$ and $l$ be nonnegative integers with $\phi_{m}\left(p^{l}-1\right) \leq p^{n}$. Let

$$
\beta_{n, m, l}=\left(\rho^{m} T^{p^{l}-1}+\sum_{k=1}^{m} \rho^{m-k} T^{\phi_{k-1}^{\prime}\left(p^{l}-1\right)-1}\right) u_{n, p}+\rho^{m+l+1} w_{n} .
$$

Then $\beta_{n, m, l} \in V_{n, \phi_{m}^{\prime}\left(p^{l}-1\right)}(-\xi)$ unless $l=n-1$ and $m=0$, in which case $\beta_{n, 0, n-1} \in$ $V_{n, p^{n}}\left(\xi^{p^{-1}}\right)$. Furthermore, for any $j \geq 0$ with $\phi_{m}(j) \leq p^{n}$, we have

$$
\left(p^{m} b T^{j}+c\right) u_{n, p}+d w_{n} \notin V_{n, \min \left(\phi_{m}^{\prime}(j)+p-1, p^{n}+p-1\right)}
$$

for all $b \in \mathbb{Z}_{p}[\Phi]-p \mathbb{Z}_{p}[\Phi], c \in T^{j+1} A_{n}$ and $d \in \mathbb{Z}_{p}[\Phi]$. 
4.3. Generating sets. In this final subsection, we turn to the task of finding small generating sets for the groups $V_{n, i}$ as $A_{n}$-modules. First, we define the refined elements that will be used in forming these sets.

Suppose that $i \leq p^{n}$ and

$$
0 \leq m \leq\left\lceil\log _{p}\left(\frac{i+1}{r+1+\delta(p-1)}\right)\right\rceil .
$$

Aside from the case that $r=p-1$ and $p^{m}<i-e_{n}<p^{m+1}$, we set $\kappa_{n, m, i}=\varphi^{n} N_{n} \kappa_{m, i}$, which can be written down explicitly as in the formulas (3.3.5), (3.3.6), and (3.3.7), but now with $u_{r}$ replaced by $u_{n, r}$ and $w$ replaced by $w_{n}$. By Propositions 3.3.9, 4.1.6 and 4.2.4, we have $\kappa_{n, m, i} \in V_{n, i}$.

If $r=p-1$ and $p^{m}<i-e_{n}<p^{m+1}$, then we set $\kappa_{n, m, i}=\omega_{n, m, m-\sigma(m+1, i)}$ with $\omega_{n, m, l}$ for $l \geq 0$ defined as in (4.2.1). Then $\kappa_{n, m, i} \in V_{i}$ by the claim in the proof of Theorem 4.2.2. Moreover, we have

$$
\kappa_{n, m, i}=\sum_{k=\sigma(m+1, i)}^{m} \rho^{k} \vartheta_{n-m, m-k} T^{\theta_{k}(i)-1} u_{n, p-1}-v,
$$

since $\theta_{k}(i)=p^{n-k-2}(p-1)+p^{m-k}$ if $k \geq \sigma(m+1, i)$.

Our next result is the analogue of Theorem 3.4.1 at the finite level.

Theorem 4.3.1. Let $\mu$ be the smallest nonnegative integer for which $i \leq \mu e_{n}+p^{n}$. Let

$$
S_{n, i}=\left\{p^{\mu} \kappa_{n, m, i-\mu e_{n}} \mid 0 \leq m \leq s\right\}, \quad \text { where } s=\left\lceil\log _{p}\left(\frac{i-\mu e_{n}+1}{r+1+\delta(p-1)}\right)\right\rceil .
$$

If $2 \leq r \leq p-2$, then the $A_{n}$-module $V_{n, i}$ is generated by $S_{n, i}$. If $r=p-1$, it is generated by $S_{n, i} \cup\left\{p^{\mu} v\right\}$ if $i \leq(\mu+1) e_{n}$ and $S_{n, i}$ otherwise, and if $r=p$, it is generated by $S_{n, i} \cup\left\{p^{\mu+\left\lceil\log _{p}\left(i-\mu e_{n}\right)\right\rceil} w_{n}\right\}$.

Proof. Suppose first that $i \leq p^{n}$. If $r \neq p-1$, then $V_{n, i}=N_{n} V_{i}$ by Proposition 4.1.6. For such $i$, the generation then follows immediately from Theorem 3.4.1.

Similarly, if $r=p-1$, then $v \in V_{n, e_{n}}(-\xi)$ generates the cokernel of $N_{n}$. If $i \leq e_{n}$, then $S_{n, i} \cup\{v\}$ generates $V_{n, i}$ by a similar argument to that given in Theorem 3.4.1 (or by Proposition 4.1.6 and Theorem 3.4.1 itself). If $e_{n}<i<p^{n}$, then similarly $S_{n, i} \cup\{p v\}$ generates $V_{n, i}$, but we now claim that $p v$ is in the $A_{n}$-submodule generated by $S_{n, i}$. To see this, suppose that $m \leq n-2$ is such that $p^{m}<i-e_{n}<p^{m+1}$. Note that $A_{n} S_{n, i}$ contains $v_{n, k, i}=\rho^{k} T^{\theta_{k}(i)} u_{n, p-1}$ for each $0 \leq k \leq n-1$. (If $\kappa_{n, k, i}$ is not this element, one can multiply it by $T$ and subtract off multiples of the $v_{n, h, i}$ for $h<k$ to reduce it to this form.) Noting (4.3.1), we have

$$
\rho v=-\rho \kappa_{n, m, i}+\sum_{k=\sigma(m+1, i)}^{m} \vartheta_{n-m, m-k} T^{\theta_{k}(i)-\theta_{k+1}(i)-1} v_{n, k+1, i} \in A_{n} S_{n, i} .
$$


In the case of arbitrary $r$ and $i$, Lemma 4.1.5 tells us that $V_{n, i}=p^{\mu} V_{n, i-\mu e_{n}}$, and we again have the desired generation.

Remark 4.3.2. For $i \leq p^{n}$, the integer $s$ in Theorem 4.3.1 is unique such that $i$ lies in the half-open interval $\left[(r+1) p^{s-1},(r+1) p^{s}\right)$ if $r \leq p-1$ and $\left[2 p^{s}, 2 p^{s+1}\right)$ if $r=p$. Since $S_{n, i}$ has $s+1$ elements, the generating set $S_{n, i}^{\prime}$ provided in Theorem 4.3.1 has at most $n+1$ elements. Since $S_{n, i}^{\prime}=p^{\mu} S_{n, i-\mu e_{n}}^{\prime}$, the latter statement holds for all $i$. In fact, for $i>p^{n-1}$, the set $S_{n, i}^{\prime}$ has either $n$ or $n+1$ elements, depending for each $r$ on which of two ranges $i$ lies in modulo $e_{n}$.

Finally, we prove a slightly weaker minimality statement than Theorem 3.4.4, since in the finite case there are many values of $i$ for which the analogous statement to Theorem 3.4.4 is simply not true, so long as $r \leq p-1$.

Theorem 4.3.3. Every generating subset of the generating set for $V_{n, i}$ of Theorem 4.3.1 is of cocardinality at most one.

Proof. We maintain the notation of Theorem 4.3.1. By Lemma 4.1.5, the $p^{\mu}$ th power map defines an isomorphism $V_{i-\mu e_{n}} \stackrel{\sim}{\longrightarrow} V_{i}$, and $S_{n, i}=p^{\mu} S_{n, i-\mu e_{n}}$. We therefore assume that $i \leq p^{n}$ for the rest of the proof. Note that we have

$$
\theta_{k}(i) \leq p^{n-k-1}
$$

for all $0 \leq k \leq n-1$, and we have $\theta_{n}(i)=0$.

Case $r \leq p-2$. In this case, $N_{n}$ induces an isomorphism $D^{(r)} / f_{n} D^{(r)} \sim D_{n}^{(r)}$, so Proposition 4.1.6 tells us that $V_{n, i} \cong V_{i} /\left(V_{i} \cap f_{n} D^{(r)}\right)$. In other words, a subset $Y_{n}$ of $S_{n, i}$ will generate $V_{n, i}$ if and only if the subset $Y$ of $S_{i}$ lifting it has the property that $Y \cup\left\{f_{n} u_{r}\right\}$ generates $V_{i}+f_{n} D^{(r)}$.

Recall that

$$
f_{n} \equiv \sum_{k=0}^{n-1} p^{k} T^{p^{n-k-1}} \bmod \left(p^{n-1} T^{2}, p^{n-2} T^{2 p}, \ldots, T^{2 p^{n-1}}\right) .
$$

Noting (4.3.2), we have

$$
f_{n} \equiv p^{m} T^{p^{n-m-1}} \bmod \left(p^{m+1}, T^{\theta_{m}(i)+1}\right)
$$

for each $0 \leq m \leq s$. Let us set $I=(p, T, \varphi-1)$ and $I_{m}=\left(p, T^{1+\epsilon_{m}(i)}, \varphi-1\right)$ for the remainder of the proof.

The analogue of (3.4.3) in our current setting is

$$
\sum_{m=0}^{s} c_{m} \kappa_{m, i}=b f_{n} u_{r}
$$


for some $c_{m} \in A$ and $b \in A$. Given a solution to (4.3.4), we claim that there exist $q_{k} \in \mathbb{Z}_{p}$ for $k \leq s$, independent of the solution, such that

$$
c_{k} \equiv q_{k} b T^{\epsilon_{k}(i)} \bmod I_{k} .
$$

Of course, only those $\kappa_{n, k, i}$ for $k$ such that $p \nmid q_{k}$ and $\epsilon_{k}(i)=0$ can possibly be $A_{n}$-linear combinations of the others. If $k$ is such a value and we suppose that $c_{k}=0$, then these congruences force $b \in I$ and therefore $c_{m} \in I$ for every other $m \leq s$, proving the result.

We turn to the proof of the claim. In our current setting, (3.4.5) becomes

$$
c_{n} \rho^{n} \equiv 0 \bmod \left(p^{n+1}, T\right)
$$

for $m=n$ (if $s=n$, since $\theta_{n}(i)=0$ ) and

$c_{m} \rho^{m} T^{\psi_{m}(i)} \equiv \sum_{k \in X_{m}} c_{k} a_{k, i} \rho^{m} T^{\theta_{m}(i)-1}+b p^{m} T^{p^{n-m-1}} \bmod \left(p^{m+1}, T^{\theta_{m}(i)+1}\right)$

for $m \leq n-1$, with $X_{m}$ as in (3.4.4). In the case that $s=n$, the claim for $k=n$ is then immediate. Moreover, supposing that we know the claim for $k$ with $m+1 \leq k \leq s$, the congruence (4.3.6) implies that

$$
c_{m} \equiv \sum_{k \in X_{m}} q_{k} a_{k, i} b T^{\epsilon_{m}(i)}+b T^{p^{n-m-1}-\psi_{m}(i)} \bmod I_{m}
$$

upon application of (4.3.5) for $k \in X_{m}$. As $\epsilon_{m}(i) \leq p^{n-m-1}-\psi_{m}(i)$ by (4.3.2), we have the claim for $k=m$ as well.

We remark that if $\theta_{m}(i)<p^{n-m-1}$ for all $m \leq n-1$, which is to say that $i \leq p^{n-1} r$, then we obtain recursively that $p \mid q_{m}$ for all $m \leq s$. In other words, $S_{n, i}$ has no proper generating subset for such $i$. This is useful in the following case.

Case $r=p$. In the case $r=p$, we have $\theta_{m}(i)<p^{n-m-1}$ for all $m \leq n-1$ and all $i \leq p^{n}$ (since $\delta=1$ ), and the analogous argument working modulo $A w$ and using the set $X_{m}^{\prime}$ of (3.4.6) shows that any subset of $S_{n, i} \cup\left\{p^{\left\lceil\log _{p}(i)\right\rceil} w_{n}\right\}$ that generates $V_{n, i}$ must contain $S_{n, i}$.

Case $r=p-1$. Finally, we consider the more subtle case that $r=p-1$. In this case, $s \leq n-1$. Recall from Theorem 4.1.7 that

$$
V_{p-1} / f_{n} V_{p-1} \cong A_{n} u_{n, p-1} \cong A_{n} /\left(N_{\Gamma_{n} \times \Phi}\right)
$$

and $A_{n} v=\mathbb{Z}_{p} v+\mathbb{Z}_{p}[\Phi] N_{\Gamma_{n}} u_{n, p-1}$. Note that $N_{\Gamma_{n}}$ lifts to $T^{-1} f_{n}$ in $A$. As in (4.3.3), we have

$$
T^{-1} f_{n} \equiv p^{m} T^{p^{n-m-1}-1} \bmod \left(p^{m+1}, T^{\theta_{m}(i)+1}\right)
$$


for $0 \leq m \leq n-2$ and

$$
T^{-1} f_{n} \equiv p^{n-1}\left(1-\frac{1}{2} T\right) \bmod \left(p^{n}, T^{2}\right) .
$$

Range $i \leq e_{n}$ : In this range, every $\kappa_{n, m, i}$ lies in $A_{n} u_{r}$, so $v$ is in particular necessary to generate $V_{n, i}$. We also have $\theta_{n-1}(i)=0$ and $\theta_{m}(i) \leq e_{n-m-1}$ for all $m \leq n-2$. Consider the following analogue of (3.4.3):

$$
\sum_{m=0}^{s} c_{m} \kappa_{m, i}=b T^{-1} f_{n} u_{p-1}
$$

As before, we claim that there exist $q_{k} \in \mathbb{Z}_{p}$ for $k \leq s$, independent of the solution to (4.3.8), such that (4.3.5) holds, from which the result follows in this range.

The analogue of (3.4.5) for $m \leq s$ in the current setting is $c_{m} \rho^{m} T^{\psi_{m}(i)}$

$$
\equiv \sum_{k \in X_{m}} c_{k} a_{k, i} \rho^{m} T^{\theta_{m}(i)-1}+b p^{m} T^{p^{n-m-1}-1} \bmod \left(p^{m+1}, T^{\theta_{m}(i)+1}\right) .
$$

If $s=n-1$, we then obtain $c_{n-1} \equiv b \bmod I$. If $s \geq n-2$, we have

$$
c_{n-2} \equiv b T^{p-1-\theta_{n-2}(i)+\epsilon_{n-2}(i)} \bmod I_{n-2},
$$

and hence the claim for $k=n-2$. For $m \leq n-3$, we have $\theta_{m}(i) \leq p^{n-m-1}-2$, and assuming the claim for $m+1 \leq k \leq s$, we see recursively using (4.3.9) that

$$
c_{m} \equiv \sum_{k \in X_{m}} q_{k} a_{k, i} b T^{\epsilon_{m}(i)} \bmod I_{m} .
$$

Range $e_{n}<i<p^{n}$. In this range, $s=n-1, \theta_{n-1}(i)=1$, and $\theta_{n-2}(i)=p$. Let $l \leq n-2$ be such that $p^{l}<i-e_{n}<p^{l+1}$, so $\kappa_{n, l, i}$ is the lone element of $S_{n, i}$ that does not lie in $A_{n} u_{n, p-1}$. Thus, if we were to have

$$
\sum_{m=0}^{n-1} d_{m} \kappa_{n, m, i}=0
$$

for some $d_{m} \in A_{n}$, then we would have to have $d_{l} \in A_{n}(T, \varphi-1)$ in order that $d_{l} \kappa_{n, l, i} \in A_{n} u_{n, p-1}$. Let

$$
\kappa_{l, i}^{\prime}=\sum_{j=\sigma(l+1, i)}^{l} \rho^{j} \vartheta_{n-l, l-j} T^{\theta_{j}(i)} u_{p-1}
$$

so that $T \kappa_{n, l, i}=\varphi^{n} N_{n} \kappa_{l, i}^{\prime}$. Let $\kappa_{m, i}^{\prime}=\kappa_{m, i}$ for $m \leq n-1$ with $m \neq l$.

Now (4.3.10) implies that

$$
\sum_{m=0}^{n-1} c_{m} \kappa_{m, i}^{\prime} \equiv b T^{-1} f_{n} u_{p-1} \bmod A(\varphi-1) u_{p-1}
$$


for some $b \in A$ and where $c_{m} \in A$ reduces to $d_{m}$ for $m \neq l$ and $c_{l} \in A$ is such that $T c_{l}$ reduces to $d_{l}$ modulo $A_{n}(\varphi-1)$. Similarly to before, we claim that there exist $q_{m} \in \mathbb{Z}_{p}$ for $m \leq n-2$, independent of the solution to (4.3.11), such that (4.3.5) holds, and that $b \in I$ if and only if $c_{n-1} \in I$. From this, it follows that a solution to (4.3.11) with $c_{k}=0$ for some $k$ has $c_{m} \in I$ for every other $m \leq n-1$.

Note that $\epsilon_{l}(i)=0$, and let $\tau_{m}$ be $\vartheta_{n-l, l-m}$ if $\sigma(l+1, i) \leq m<l$ and 0 otherwise. Equations (4.3.7) and (4.3.11) yield

$$
c_{n-1} \equiv b\left(1-\frac{1}{2} T\right) \bmod \left(p, T^{2}, \varphi-1\right),
$$

and, for arbitrary $m \leq n-2$, we have

$c_{m} T^{\psi_{m}(i)}$
$\equiv \sum_{k \in X_{m}} c_{k} a_{k, i} T^{\theta_{m}(i)-1}-\tau_{m} c_{l} T^{\theta_{m}(i)}+b T^{p^{n-m-1}-1} \bmod \left(p, T^{\theta_{m}(i)+1}, \varphi-1\right)$.

For $m=n-2$, note that (4.3.12), (4.3.13), and $a_{n-1, i}=-1$ imply that

$$
c_{n-2} T^{1-\epsilon_{n-2}(i)} \equiv b-c_{n-1} \equiv \frac{1}{2} b T \bmod \left(p, T^{2}, \varphi-1\right),
$$

so (4.3.5) holds with $q_{n-2}=\frac{1}{2}$. For $m$ with $\sigma(n-1, i) \leq m \leq n-3$ (which exists only if $l=n-2)$, we have $X_{m}=\{n-1\}$ and $\theta_{m}(i)=p^{n-m-1}$, and we obtain from (4.3.13) and (4.3.14) that

$$
\begin{aligned}
c_{m} T^{1-\epsilon_{m}(i)} & \equiv-c_{n-1}-c_{n-2} \tau_{m} T+b \\
& \equiv \frac{1}{2}\left(1-\vartheta_{2, n-m-2}\right) b T \bmod \left(p, T^{2}, \varphi-1\right),
\end{aligned}
$$

so (4.3.5) holds with $q_{m}=-\frac{1}{2}(n-m-2)$. For $m<\sigma(n-1, i)$, we have $\theta_{m}(i)<p^{n-m-1}$, and (4.3.13) and (4.3.14) yield recursively that

$$
c_{m} \equiv \sum_{k \in X_{m}} q_{k} a_{k, i} b T^{\epsilon_{m}(i)}-q_{l} \tau_{m} b T^{\epsilon_{m}(i)}+b T^{p^{n-m-1}-1-\psi_{m}(i)} \bmod I_{m},
$$

verifying (4.3.5) for $k=m$.

\section{Acknowledgments}

The idea for this paper originated with my $1999 \mathrm{PhD}$ thesis, and initial computations were performed on an evening in June 2001 during a visit to the University of Nottingham. I thank Ivan Fesenko for his hospitality. I wrote a short draft of the paper in 2002 and made additions to it in August 2006. The paper tripled in size as I brought it to near final form in the summer of 2011. I also thank Richard Gottesman for his interest in this work, which inspired me to finish the paper. 


\section{References}

[Greither 1996] C. Greither, "On Chinburg's second conjecture for abelian fields", J. Reine Angew. Math. 479 (1996), 1-37. MR 97e:11139 Zbl 0856.11051

[Serre 1979] J.-P. Serre, Local fields, Graduate Texts in Mathematics 67, Springer, New York, 1979. MR 82e:12016 Zbl 0423.12016

[Sharifi 2002] R. T. Sharifi, "Determination of conductors from Galois module structure", Math. Z. 241:2 (2002), 227-245. MR 2003h:11147 Zbl 1017.11058

[Wintenberger 1983] J.-P. Wintenberger, "Le corps des normes de certaines extensions infinies de corps locaux; applications", Ann. Sci. École Norm. Sup. (4) 16:1 (1983), 59-89. MR 85e:11098 Zbl 0516.12015

Communicated by John $\mathrm{H}$. Coates

Received 2011-08-20 Revised 2011-11-29 Accepted 2012-02-20

sharifi@math.arizona.edu Department of Mathematics, University of Arizona, 617 N. Santa Rita Ave, PO Box 210089,

Tucson AZ 85721-0089, United States

http://math.arizona.edu/ sharifi 


\section{Algebra \& Number Theory}

msp.org/ant

\section{EDITORS}

MANAGING EDITOR

Bjorn Poonen

Massachusetts Institute of Technology

Cambridge, USA

\author{
EDITORIAL BOARD CHAIR \\ David Eisenbud \\ University of California \\ Berkeley, USA
}

\section{BOARD OF EDITORS}

Georgia Benkart

Dave Benson

Richard E. Borcherds

John H. Coates

J-L. Colliot-Thélène

Brian D. Conrad

Hélène Esnault

Hubert Flenner

Edward Frenkel

Andrew Granville

Joseph Gubeladze

Ehud Hrushovski

Craig Huneke

Mikhail Kapranov

Yujiro Kawamata

János Kollár

Yuri Manin

Barry Mazur

Philippe Michel
University of Wisconsin, Madison, USA

University of Aberdeen, Scotland

University of California, Berkeley, USA

University of Cambridge, UK

CNRS, Université Paris-Sud, France

University of Michigan, USA

Freie Universität Berlin, Germany

Ruhr-Universität, Germany

University of California, Berkeley, USA

Université de Montréal, Canada

San Francisco State University, USA

Hebrew University, Israel

University of Virginia, USA

Yale University, USA

University of Tokyo, Japan

Princeton University, USA

Northwestern University, USA

Harvard University, USA

École Polytechnique Fédérale de Lausanne
Susan Montgomery

Shigefumi Mori

Raman Parimala

Jonathan Pila

Victor Reiner

Karl Rubin

Peter Sarnak

Joseph H. Silverman

Michael Singer

Vasudevan Srinivas

J. Toby Stafford

Bernd Sturmfels

Richard Taylor

Ravi Vakil

Michel van den Bergh

Marie-France Vignéras

Kei-Ichi Watanabe

Andrei Zelevinsky

Efim Zelmanov
University of Southern California, USA

RIMS, Kyoto University, Japan

Emory University, USA

University of Oxford, UK

University of Minnesota, USA

University of California, Irvine, USA

Princeton University, USA

Brown University, USA

North Carolina State University, USA

Tata Inst. of Fund. Research, India

University of Michigan, USA

University of California, Berkeley, USA

Harvard University, USA

Stanford University, USA

Hasselt University, Belgium

Université Paris VII, France

Nihon University, Japan

Northeastern University, USA

University of California, San Diego, USA

\section{PRODUCTION}

production@msp.org

Silvio Levy, Scientific Editor

See inside back cover or msp.org/ant for submission instructions.

The subscription price for 2013 is US \$200/year for the electronic version, and \$350/year ( $\$ 40$, if shipping outside the US) for print and electronic. Subscriptions, requests for back issues and changes of subscribers address should be sent to MSP.

Algebra \& Number Theory (ISSN 1944-7833 electronic, 1937-0652 printed) at Mathematical Sciences Publishers, 798 Evans Hall \#3840, c/o University of California, Berkeley, CA 94720-3840 is published continuously online. Periodical rate postage paid at Berkeley, CA 94704, and additional mailing offices.

ANT peer review and production are managed by EditFLOW ${ }^{\circledR}$ from Mathematical Sciences Publishers.

\section{PUBLISHED BY}

mathematical sciences publishers

nonprofit scientific publishing

http://msp.org/

(C) 2013 Mathematical Sciences Publishers 


\section{Algebra \& Number Theory}

\section{Volume $7 \quad$ No. $1 \quad 2013$}

Powers of ideals and the cohomology of stalks and fibers of morphisms

MARC CHARDIN

Graphs of Hecke operators

OLIVER LORSCHEID

Group actions of prime order on local normal rings

FrANZ KIRÀLY and WERNER LÜTKEBOHMERT

On the arithmetic and geometry of binary Hamiltonian forms

JOUNI PARKKONEN and FRÉDÉRIC PAULIN

$L$-functions and periods of adjoint motives

MICHAEL HARRIS

Galois module structure of local unit groups

ROMYAR SHARIFI

On the invariant theory for tame tilted algebras

CALIN CHINDRIS

Period functions and cotangent sums

SANDRO BETTIN and BRIAN CONREY 